\title{
Aggregation functions for typical hesitant fuzzy elements and the action of automorphisms
}

\author{
Benjamin Bedregal $^{\mathrm{a}, *}$, Renata Reiser ${ }^{\mathrm{b}}$, Humberto Bustince ${ }^{\mathrm{c}}$, Carlos Lopez-Molina ${ }^{\mathrm{c}}$, \\ Vicenç Torra ${ }^{\mathrm{d}}$ \\ ${ }^{a}$ Department of Informatics and Applied Mathematics, Federal University of Rio Grande do Norte, Natal, Brazil \\ ${ }^{\mathrm{b}}$ Center of Technological Development, Federal University of Pelotas, Pelotas, Brazil \\ ${ }^{\mathrm{c}}$ Departamento de Automática y Computación, Universidad Pública de Navarra, Campus Arrosadia s/n, P.O. Box 31006, Pamplona, Spain \\ ${ }^{\mathrm{d}}$ Inst. de Investigación en Inteligencia Artificial, Cons. Superior de Investigaciones Científicas, Campus de la Universidad Autonoma de Barcelona s/n, P.O. \\ Box 08193, Barcelona, Spain
}

\section{A R T I C L E I N F O}

\section{Article history:}

Received 4 October 2012

Received in revised form 3 August 2013

Accepted 13 August 2013

Available online 27 August 2013

\section{Keywords:}

Hesitant fuzzy set

Automorphism

Order

Representability

ษ-representation

Aggregation operator

\begin{abstract}
A B S T R A C T
This work studies the aggregation operators on the set of all possible membership degrees

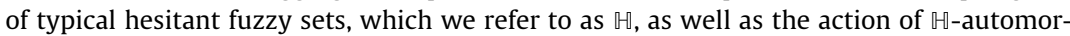
phisms which are defined over the set of all finite non-empty subsets of the unitary interval. In order to do so, the partial order $\leqslant_{\mathbb{H}}$, based on $\alpha$-normalization, is introduced, leading to a comparison based on selecting the greatest membership degrees of the related fuzzy sets. Additionally, the idea of interval representation is extended to the context of typical hesitant aggregation functions named as the $\mathbb{\sharp}$-representation. As main contribution, we consider the class of finite hesitant triangular norms, studying their properties and analyzing the $\mathbb{-}$-conjugate functions over such operators.
\end{abstract}

(C) 2013 Elsevier Inc. All rights reserved.

\section{Introduction}

The fuzzy set theory was introduced as a mathematical framework to deal with the incompleteness of information of real systems and the necessity of combining granularity and flexibility in the representation of such information in practical reasoning tasks. Since first introduced by Zadeh [44], many extensions of the fuzzy set theory have been conceived.

Type-2 Fuzzy Sets (T2FSs) are an important generalization of classical fuzzy sets able to model vague concepts via more flexible (non-precise) membership functions. Some of the main results on this generalization are summarized in [18,19]. Their flexibility in the representation of the ambiguity comes coupled to severe problems in their practical applications. Interval-valued fuzzy sets (IVFSs) were conceived [30,45] as a particular class of T2FSs which captures the imprecision of the membership degree as an interval, reflecting the measure of vagueness and uncertainty in the width of such intervals. Further significant results are also Atanassov's intuitionistic fuzzy sets [1] (AIFSs), taking into account concepts of intuitionistic logic by considering the hesitation related to the dual construction of (non-)membership degrees. See more details in $[2,4]$. An integrated approach, named as the interval-valued intuitionistic fuzzy set theory [3] is born from the combination of the concept of IVFS and AIFS by relaxing the complementary operation and modelling the membership degrees by means

\footnotetext{
* Corresponding author. Tel.: +55 84 32153814; fax: +55 8432153813 .

E-mail addresses: bedregal@dimap.ufrn.br (B. Bedregal), reiser@inf.ufpel.edu.br (R. Reiser), bustince@unavarra.es (H. Bustince), carlos.lopez@unavarra.es (C. Lopez-Molina), vtorra@iiia.csic.es (V. Torra).
} 
of intervals or Atanassov's intuitionistic pairs. Another direction of research, but with analogous results, considers vague sets (see, e.g., $[8,22])$.

On the hall of approaches related to many-valued fuzzy sets, this work focuses on the study of Hesitant Fuzzy Logic (HFL) whose foundations come from the theory of Hesitant Fuzzy Sets (HFSs). This theory was recently introduced in [33,34] as an appropriate tool to deal with multi-criteria decision making. Thus, it would be possible to take a set of values grouped together based on certain criteria, in order to define the membership degree of an element in a HFS.

\subsection{Related works on aggregating information based on HFSs}

Very frequently, distinct approaches to deal with multi-criteria decision making use aggregation operators to group the information prior to the reasoning phase. These operators have an important role in fuzzy reasoning, as presented in $[10,11,15,17,18,46,47]$. Very relevant examples of aggregation operators often used for decision making are the Weighted Averaging (WA) and Ordered Weighted Averaging (OWA) operators, which are based on weighting vectors, and their corresponding continuous extensions C-WA and C-OWA [43]. These operators have been subsequently generalized to produce aggregated information based on confidence indexes and weighting vectors, giving rise to Confidence Induced Weighted Aggregation (CIWA) and Confidence Induced Ordered Weighted Aggregation (CIOWA) operators [38] (a review of such operators is included in Section 2).

Moreover, different versions of these operators have been proposed thereafter to aggregate information provided as nonclassical fuzzy sets. By using these operators, decision making algorithms are able to deal with different representations of uncertainty. Regarding HFSs, Xia et al. propose a series of such aggregation operators so that they can be used in situations in which there exist difficulties in expressing the membership degree of an element as a scalar value. Further studies [40,42] have produced a wide family of aggregation operators and concepts of entropy and cross-entropy for hesitant fuzzy information are discussed including their desirable properties. In [13], some correlation coefficient formulas for HFSs are derived and applied to clustering analysis under hesitant fuzzy environments. In [48], extensions for the hesitant context of the Bonferroni mean and some of their variants were proposed and applied in multi-criteria decision making.

Research on HFSs has also explored the application of aggregation operators with purposes other than WA operators. In [28], the concept of hesitant fuzzy linguistic term set was introduced to manage hesitation in qualitative contexts and applied in group making decision in [29]. Interesting results related to distances and other similar measures for HFSs are presented in [41]. Additionally, in [37], aggregation operators for HFSs were introduced along with the relationship between HFSs and AIFSs. In [20], a mutual transformation of the entropy into the similarity measure for HFSs was proposed and a partial order on HFSs based on the normalization of hesitant fuzzy elements was also proposed in [41].

More recently, aggregation operators defined over HFSs and their application to multiple attribute decision making are studied in [26,35,36,39]. Furthermore, interval-valued hesitant preference relations were introduced in [12] describing uncertain evaluation information in group decision making processes.

\subsection{Relevance of new aggregation functions for HFSs by integration of formal concepts from Fuzzy Logic and Lattice Theory}

It is well known that the information provided by inference systems modelled by the Fuzzy Logic and founded on the Fuzzy Set Theory can be formally discussed and compared in terms of the partial ordered sets defined in accordance with the Lattice Theory. In lattice-valued fuzzy set theory, aggregation functions are increasing operators with respect to the order of the lattice [24,25].

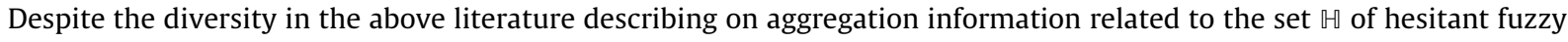
sets (HFSs), except the recent results presented in [20] dealing with a partial order relation on HFSs, the major contributions have considered only relations based on distance or score functions to order the hesitant fuzzy elements. As one can easily notice, they are not partial orders since there exist at least two hesitant fuzzy elements associated to the same score image. Moreover, these approaches cannot provide an explicit definition of a hesitant aggregation function (HAF) on $\mathbb{H}$. Consequently, they can be applied to many distinct scenarios but they are only able to specify aggregation operators on $₫$ which extend some well known aggregation function on $[0,1]$.

Differently from such literature which define specific HAFs even without a formal concept of an HAF, in this paper the definition of HAFs is consistent with the definition of aggregation functions valued in the complete bounded lattice $\left(\mathbb{H}, \leqslant_{\mathbb{H}}\right)$, whenever the partial order $\leqslant_{\mathbb{H}}$ is fixed according to [24,25]. As the main benefit, the minimum criteria for a multidimensional HFA $F: \mathbb{Q}^{n} \rightarrow \mathbb{U}$ can be formalized.

Founded on the Lattice Theory, distinct ways to obtain partial ordered HFSs on $\mathbb{H}$ are presented, which are based on two general normalization principles. By fixing one of this normalization and defining a binary relation $\leqslant_{\mathbb{H}}$ as the more intuitive partial order on $\mathbb{H}$, we are able to compare (by reporting to the usual order on $[0,1]$ ) one by one all the corresponding most relevant elements of a compatible pair of normalized hesitant fuzzy sets. So, when an HAF is formally defined according to the bounded lattice-valued fuzzy aggregation functions, as considered in [24], we obtain the definition of a hesitant t-norm in $\left(\mathbb{H}, \leqslant_{\mathbb{H}}\right)$ consistently with the definition of a valued t-norm in $([0,1], \leqslant)$. Inspired by the OWA-like operators, many operators have been defined for the context of HFS theory. In addition, four classes of OWAs are reported in order to show that OWA operators can also be defined on the lattice $\left(\mathbb{H}, \leqslant_{\mathbb{H}}\right)$. 


\subsection{Main purposes of this work}

This paper studies the aggregation operators for the class of Typical Hesitant Fuzzy Sets (THFSs), i.e. for those fuzzy sets defined over the set $\mathbb{H} \subseteq \wp([0,1])$ of all finite non-empty subsets of the unitary interval $[0,1]$. Additionally, we introduce a novel partial order $\leqslant_{H}$, which is based on $\sigma$-permutation followed by $\alpha$-normalization. Consequently, it is possible to discuss the monotonous information aggregation between typical hesitant fuzzy elements with respect to the complete bounded lattice $(\mathbb{H}, \leqslant \mathbb{H})$.

The notion of Typical Hesitant Fuzzy Aggregation Function (THAF) on the lattice $\left(\mathbb{H}, \leqslant_{\mathbb{H}}\right)$ extends the notion of fuzzy Aggregation Function (AF) on the usual lattice $([0,1], \leqslant)$. This notion of THAF is compatible with the one introduced in $[11,24,25]$, connecting the general concept of AF on bounded partially ordered sets and that on bounded lattices. Moreover, it is also consistent with the order information performed on $\mathcal{L}^{I}$, i.e. on the set of all the closed subintervals of the unit interval $[0,1]$, well-known as the underlying lattice of interval-valued fuzzy set theory (or equivalently, of Atanassov's intuitionistic fuzzy set theory) [15]. This work provides reasonable criteria to guarantee the (strict) isotonicity of THAFs (mainly related to t-norms) which is compatible with the corresponding logical construction on THFSs, what constitutes a significant advantage in using the partial order on $\left(\mathbb{H}, \leqslant_{\mathbb{H}}\right)$.

As main contribution of this work, we propose two natural methodologies to produce THAFs from AFs, in accordance to $\left(\mathbb{H}, \leqslant_{\mathbb{H}}\right)$. Thus, by using an ordered aggregation operator $M$ as AF we can define two ordered aggregation operators $\widehat{M}$ and $\widetilde{M}$ as THAF from such methodologies, meaning that more relevant information can be considered from the partial order $\leqslant_{\mathbb{H}}$.

In addition to the construction method for THAFs from AFs, we introduce in this paper the Finite Hesitant Triangular

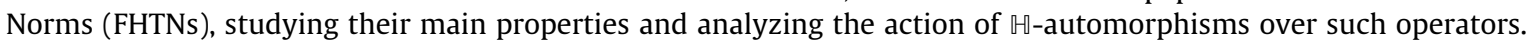

\subsection{Paper outline}

The paper is organized as follows. After the preliminaries in Section 2, THFSs are defined in Section 3 as subsets of finite and non-empty fuzzy sets of the unitary interval. Section 3 includes as well the definition of $\alpha$-normalization over finite HFSs to compare the values in THFSs. In Section 4, two natural methodologies to obtain THAFs from AFs are considered,

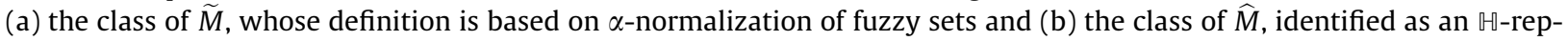
resentation of a given aggregation function $M$. The main properties of AFs, as well as the conditions under which such properties are preserved in both classes of THAFs are also discussed, particularly those related to FHTNs. Section 6 introduces the $\llbracket$-automorphisms analyzing the THAFs obtained as conjugate functions by action of such automorphisms in the class of FHTNs, including a description of conditions under which they verify the main properties of THAFs. Finally, Section 7 reports the main results and further work.

\section{Preliminaries}

In this section, we review basic concepts of aggregation functions on the unitary interval $[0,1]$, their main properties and examples, including the important class of triangular norms. We also recall the definition of an automorphism on [0,1].

Definition 1 [9, Definition 0]. An automorphism is a function $\phi:[0,1] \rightarrow[0,1]$ which is bijective and increasing. Notice that, each automorphism $\phi$ is continuous, strictly increasing and $\phi(0)=0$ and $\phi(1)=1$.

Definition 2 [11, Definition 1]. An aggregation function of dimension $n$ ( $n$-ary aggregation function) is an increasing function $M:[0,1]^{n} \rightarrow[0,1]$ such that $M(0, \ldots, 0)=0$ and $M(1, \ldots, 1)=1$.

Definition 3 [11, Definitions 2 and 3]. Let $M:[0,1]^{n} \rightarrow[0,1]$ be an $n$-ary aggregation function.

(i) $M$ is said to have an annihilator $a \in[0,1]$ if $M\left(x_{1}, \ldots, x_{n}\right)=a$ whenever $a \in\left\{x_{1}, \ldots, x_{n}\right\}$.

(ii) $M$ is said to be strictly increasing if it is strictly increasing as a real function on $[0,1]^{n}$, if $M$ has no annihilator, or if it is strictly increasing in $([0,1] \backslash\{a\})^{n}$ if $a$ is an annihilator of $M$.

(iii) $M$ is said to have divisors of zero if there exist $\left.\left.x_{1}, \ldots, x_{n} \in\right] 0,1\right]$ such that $M\left(x_{1}, \ldots, x_{n}\right)=0$.

(iv) $M$ is said to be idempotent if $M(x, \ldots, x)=x$ for any $x \in[0,1]$.

For the particular case of binary AFs we recall the following definitions.

Definition 4 [11, Definition 2]. Let $M$ be a binary aggregation function.

(i) $M$ is said to be symmetric if $M(x, y)=M(y, x)$ for any $x, y \in[0,1]$.

(ii) $M$ is said to be associative if $M(M(x, y), z)=M(x, M(y, z))$ for any $x, y, z \in[0,1]$. 
Example 1. Let $\left(w_{1}, w_{2}, \ldots, w_{n}\right) \in[0,1]^{n}$ be a non-negative weighting vector such that $\sum_{i=1}^{n} w_{i}=1$. Additionally, let $\sigma$ be a permutation such that $x_{\sigma(i)}$ is the $i$ th greatest element $x_{i}$. Let $l_{i} \in[0,1]$ be the confidence level associated with each $x_{i}$. For all $\left(x_{1-}\right.$ $\left., x_{2}, \ldots, x_{n}\right) \in[0,1]^{n}$, we have the following definitions:

1. Weighted Averaging Operator, WA: $[0,1]^{n} \rightarrow[0,1]$ given by

$$
\mathrm{WA}\left(x_{1}, x_{2}, \ldots, x_{n}\right)=\sum_{i=1}^{n} w_{i} \cdot x_{i}
$$

is one of the most fundamental aggregation operators and has been applied in many areas, especially in those related to multi-attribute decision making.

2. Confidence Induced Weighted Aggregation Operator, CIWA: $[0,1]^{n} \rightarrow[0,1]$, given by

$$
\operatorname{CIWA}\left(x_{1}, x_{2}, \ldots, x_{n}\right)=\sum_{i=1}^{n} w_{i} \cdot l_{i} \cdot x_{i},
$$

was introduced in [38] and extends the WA in order to also consider the confidence level related to the familiarity of the expert with the professional field.

3. Ordered Weighted Averaging Operator, OWA: $[0,1]^{n} \rightarrow[0,1]$ given by

$$
\operatorname{OWA}\left(x_{1}, x_{2}, \ldots, x_{n}\right)=\sum_{i=1}^{n} w_{i} \cdot x_{\sigma(i)},
$$

was originally introduced by Yager in [43] to aggregate scores associated with the satisfaction of multiple criteria, in which the aggregated arguments are rearranged in descending order, and the weight vector is merely associated with their ordered positions.

4. Confidence Induced Ordered Weighted Averaging Operator, CIOWA: $[0,1]^{n} \rightarrow[0,1]$ given by

$$
\operatorname{CIOWA}\left(x_{1}, x_{2}, \ldots, x_{n}\right)=\sum_{i=1}^{n} w_{i} \cdot l_{\sigma(i)} \cdot x_{\sigma(i)},
$$

provides a parameterized family of aggregation operators, which includes many of the well-known operators such as: (i) the minimum operator when $w_{1}=1$ and $w_{j}=0$ for $j \neq 1$; (ii) the maximum operator when $w_{n}=1$ and $w_{j}=0$ for $j \neq n$; and (iii) the arithmetic median operator when $w_{j}=\frac{1}{n}$. For more details, see [38].

We recall now the concept of t-norm which plays a key role, to model conjunctions in Fuzzy Logic or intersections in fuzzy set theory.

Definition 5 [23, Definition 2.2]. A triangular norm (t-norm in short) is an associative, symmetric and binary aggregation function $T:[0,1]^{2} \rightarrow[0,1]$ such that $T(1, x)=x$ for all $x \in[0,1]$. A strictly increasing continuous t-norm is called a strict t-norm.

A particular type of continuous t-norms is that of the Archimedean t-norms (see, e.g., [23]).

Definition 6 [23, Definition 6.7]. A continuous t-norm $T$ is said to be Archimedean if $T(x, x)<x$ for all $x \in] 0,1[$.

It is worth to remark that the one above is not the usual definition of Archimedean t-norm in the literature. Nevertheless, both definitions are equivalent when dealing with continuous t-norms ([23]). Any strict t-norm (i.e., any continuous and strictly increasing t-norm) is necessarily an Archimedean t-norm.

The following result, already presented in [23,32], shows that any strict t-norm is just the image of the conjugate function of the product t-norm $T_{P}(x, y)=x \cdot y$.

Theorem 1. A t-norm $T$ is strict if and only if there exists an automorphism $\varphi$ on the unitary interval such that

$$
T(x, y)=\varphi^{-1}(\varphi(x) \cdot \varphi(y)), \quad \forall x, y \in[0,1] .
$$

\section{Hesitant fuzzy sets}

According to [41], a Hesitant Fuzzy Element (HFE) is an element of $\wp([0,1])$, the powerset of unitary interval [0,1]. By restricting HFSs, this section introduces the complete bounded lattice $\left(\mathbb{H}, \leqslant_{\mathbb{H}}\right)$, where $\mathbb{M}$ denotes the set of Typical Hesitant Fuzzy Elements (THFEs), i.e. the subset of finite and nonempty HFEs and $\leqslant_{\mathbb{H}}$ indicates the corresponding partial order on $\mathbb{H}$.

Definition 7 [34]. Let $\wp([0,1])$ be the set of all subsets of the unitary interval and $U$ be a nonempty set. Let $\mu_{A}: U \rightarrow \wp([0,1])$, then a Hesitant Fuzzy Set (HFS) $A$ defined over $U$ is given by

$$
A=\left\{\left(x, \mu_{A}(x)\right): x \in U\right\} .
$$


Although a HFE could be any subset of $[0,1]$, practical works dealing with hesitant fuzzy sets frequently restrict to finite sets [40,41]. Notice that, if for some $x \in U, \mu_{A}(x)=\emptyset$ then $A$ makes no sense [34]. Other works on HFSs also assume explicitly or implicitly that the memberships of HFSs are finite and nonempty subsets (see this, e. g. in $[21,37,41,47]$ ).

This paper considers finite and nonempty HFSs. This is formally presented in the next definition.

Definition 8. Let $\mathbb{W} \subseteq \wp([0,1])$ be the set of all finite non-empty subsets of the interval $[0,1]$, and let $U$ be a non-empty set. A Typical Hesitant Fuzzy Set (THFS) $A$ over $U$ is given by Eq. (5), where $\mu_{A}: U \rightarrow \mathbb{H}$.

Each $X \in \mathbb{H}$ is called a Typical Hesitant Fuzzy Element of $\mathbb{H}$ (THFE) and the number of its elements, i.e. the cardinality of $X$, is referred to as $\# X$.

The set of all unitary subsets on $\wp([0,1])$ is called the set of diagonal or degenerate elements of $\llbracket$ and is denoted by $\mathcal{D}_{\sharp}$, i.e. $\mathcal{D}_{\sharp}=\{X \in \mathbb{U}: \# X=1\}$. In addition, interval-valued and Atanassov's intuitionistic fuzzy values as well as the diagonal elements of $\mathbb{H}$ are all seen as fuzzy values and they can be seen as THFSs.

\subsection{Partial orders on $₫$}

We start this section showing that the usual inclusion order $\subseteq$, restricted to the set $\llbracket \subseteq \wp([0,1])$, it is not suitable to com-

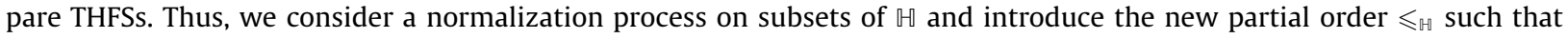
$\left(\mathbb{H}, \leqslant_{\mathbb{H}}\right)$ is a complete bounded lattice. Particularly, there are two opposite principles for such normalization:

(i) $\alpha$-normalization, by removing elements of the set having more elements, and

(ii) $\beta$-normalization, by adding elements to the set with a lower number of elements.

Both principles are described in the following sections. In particular, the $\beta$-normalization principle was also suggested in [41] (see also $[20,39,47])$.

\subsubsection{The complete bounded lattice $(\mathbb{H}, \subseteq)$}

Clearly, $(\mathbb{M}, \subseteq)$ is a complete bounded lattice. However the inclusion relation $\subseteq$ is not a suitable partial order for THFEs since it does not match the usual order on $[0,1]$ when dealing with diagonal elements, i.e. $\left(\mathcal{D}_{\mathbb{H}}, \subseteq\right) \nsucceq([0,1], \leqslant)$. Therefore, it should only be used as an auxiliary order on $\mathbb{H}$, similarly to what usually happens in interval-valued fuzzy set theory, where the main order is the product order (see $[5,14,31]$ ).

\subsubsection{The complete bounded lattice $(\mathbb{H}, \leqslant \mathbb{})$}

In order to compare two arbitrary THFEs with a partial order on $\mathbb{\sharp}$, we first consider applying normalization for them to have the same cardinality. Then, the comparison is performed by confronting one by one the values of the normalized THFEs.

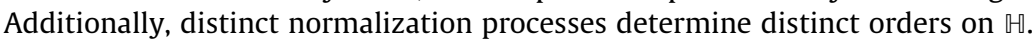

When $\mathbb{N}_{n}=\{1, \ldots, n\}$, for all $X \in \mathbb{M}$, a mapping $\sigma_{X}: \mathbb{N}_{\# X} \rightarrow X$ defines a permutation such that, for any $i \in \mathbb{N}_{\# X-1}$, we have that

$$
\sigma_{X}(i)<\sigma_{X}(i+1)
$$

That is, for any $X, Y \in \mathbb{H}^{(m)}=\{X \in \mathbb{U}: \# X=m\}$, with $m \in \mathbb{N}$, we have that

$$
X \leqslant_{\mathbb{H}^{(m)}} Y \text { iff } \sigma_{X}(i) \leqslant \sigma_{Y}(i) \text { for each } i \in \mathbb{N}_{m} .
$$

Now, the two normalization processes are defined:

(i) Let $\wp^{(m)}(X)=\left\{A \subseteq X\right.$ : \#A=m\}. Given $n \in \mathbb{N}, m \leqslant n$ and a family $\left(A_{m}\right)_{m \in \mathbb{N}_{n}}$ of sets such that $A_{m} \in \wp^{(m)}\left(\mathbb{N}_{n}\right)$, with \# $A_{m}=m$. The $\alpha$-normalization determined by $\left(A_{m}\right)_{m \in \mathbb{N}_{n}}$ is obtained by the function $\alpha: \mathbb{M} \times \mathbb{N} \rightarrow \mathbb{W}$ whose definition is given by

$$
\alpha(X, k)= \begin{cases}X, & \text { if } \# X \leqslant k \\ \left\{\sigma_{X}(i): i \in A_{k}\right\}, & \text { otherwise. }\end{cases}
$$

And, based on the $\alpha$-normalization stated by Eq. (7), it is possible to choose, for each $m \in \mathbb{N}$, the $m$ greatest elements of $X$. So, we obtained the normalized set $\alpha(X, m) \in \wp^{(m)}(X)$ determined by $A_{m}=\{n-m+1, n-m+2, \ldots, n\}$.

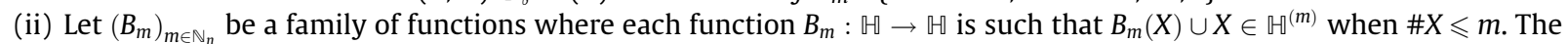
$\beta$-normalization determined by $\left(B_{m}\right)_{m \in \mathbb{N}_{n}}$ is obtained by the function $\beta: \mathbb{W} \times \mathbb{N} \rightarrow \mathbb{M}$ given by

$$
\beta(X, k)= \begin{cases}X, & \text { if } \# X \geqslant k ; \\ X \cup B_{k}(X), & \text { otherwise. }\end{cases}
$$

Let $\gamma$ be a $(\alpha-) \beta$-normalization function. Given two sets $X, Y \in \mathbb{U}$ the sets $\gamma\left(X\right.$,\#Y) and $\gamma\left(Y\right.$, \#X) belong to $\mathbb{q}^{(\min (\# X, \# Y))}$, i.e. they have the same cardinality. 
From now on we consider only $\alpha$-normalization, since it is reasonable to our main proposal. In particular, given two THFSs $X$ and $Y$, we will always consider in their $\alpha$-normalization $n=\max (\# X, \# Y), m=\min \quad(\# X$, \#Y) and $A_{m}=\{n-m+1, n-m+2, \ldots, n\}$.

Example 2. Let $X=\{0.4,0.5,0.6\}, Y=\{0.1,0.5,0.6,0.7\} \in \mathbb{H}$. Then $m=3, n=4$ and therefore $A_{3}=\{2,3,4\}$. In this conditions we obtain that $\alpha(X, \bar{Y})=\alpha(X, 4)=X$ and $\alpha(Y, \bar{X})=\alpha(Y, 3)=\left\{\sigma_{Y}(i): i \in A_{3}\right\}=\{0.5,0.6,0.7\}$. Therefore $\alpha(X, \overline{\bar{Y}})<_{\mathbb{H}^{(3)}} \alpha(Y, \overline{\bar{X}})$. So, by the $\alpha$-normalization we obtain two sets with the same cardinality $(m)$ which allows us to compare them using the strict partial order $<_{\mathbb{H} \mathbb{H}^{(m)}}$.

Additionally, by normalizing, it is possible to extend the order on $\mathbb{\sharp}^{(m)}$ in Eq. (6) for $\mathbb{\sharp}$. For instance, if we consider the $\alpha$-normalization then, for $X, Y \in \mathbb{H}$, we have that

$$
X \leqslant_{\mathbb{H}} Y \operatorname{iff}\left(\alpha(X, \# Y)<_{\mathbb{H}(m)} \alpha(Y, \# X)\right) \text { or }(\alpha(X, \# Y)=\alpha(Y, \# X) \text { and } \# Y \leqslant \# X) .
$$

Thus, according to Eq. (9), the following holds:

(i) if $m=\# X<\# Y$ then $X \leqslant_{\mathbb{H}} Y$ iff $X \leqslant_{\mathbb{H}^{(m)}} \alpha(Y, m)$;

(ii) otherwise, if $m=\# Y \leqslant \# X$, we can conclude that $X \leqslant \mathbb{H}_{\mathbb{H}} Y$ iff $\alpha(X, m)<_{\mathbb{H}^{(m)}} Y$.

Example 3. Consider $X=\{0.6,0.3,0.8\}, Y=\{0.8,0.5\} \in \mathbb{H}$. By Eqs. (7) and (9), since $m=\min (\# X$,\#Y)=2, for all $i \in\{1,2\}$ it holds that $\sigma_{\alpha(Y, 3)}(i) \leqslant \sigma_{\alpha(X, 2)}(i)$, meaning that $Y \leqslant_{\mathbb{H}^{2}} \alpha(X, 2)=\{0.6,0.8\}$. Therefore, we obtain that $X \geqslant_{\mathbb{H}} Y$.

Remark 1. The score function is frequently applied in recent works, see e.g. [26,39], in order to deal with ordered aggregation operators in HFL. In this paper, we provide another option to compare HFSs which is compatible with the partial order $\leqslant_{\mathbb{H}}$. For instance, let $X=\{0.6,0.3,0.8\}, Y=\{0.8,0.5\} \in \mathbb{H}$. According to [40], Definition 2, based on the score function $s: \mathbb{H}, \rightarrow[0,1]$, we have that $X \prec Y$ since $s(X)=\frac{1}{3}(0.6+0.3+0.8) \geqslant s \frac{1}{2}(0.8+0.5)=s(Y)$, meaning that $X$ is superior to $Y$. ${ }^{1}$ However, based on the bounded lattice $\left(\mathbb{H}, \leqslant_{\mathbb{H}}\right)$ and by Example 3 , it is immediate than $Y \leqslant_{\mathbb{H}} X$. Thus, the interpretation of the partial order $\leqslant_{\mathbb{N}}$ considers the most significant corresponding elements, by the $\alpha$-normalization and $\sigma$-permutation, when $X$ and $Y$ are compared.

There exist other possibilities to compare THFEs which are not based on normalization. For $X, Y \in \mathbb{U}$,

(i) when $m=\min (\# X$,\#Y), we have that

$$
X \unlhd_{\mathbb{H}} Y \text { iff }(X=Y) \text { or }\left(\exists i \in \mathbb{N}_{m}, \sigma_{X}(i)<\sigma_{Y}(i) \text { and } \forall j<i, \sigma_{X}(j)=\sigma_{Y}(j)\right) ;
$$

(ii) alternatively, we have

$$
X \preccurlyeq_{\mathbb{H}} Y \text { iff }\left(\frac{1}{\# X} \sum_{x \in X} x<\frac{1}{\# Y} \sum_{y \in Y} y\right) \text { or }\left(\frac{1}{\# X} \sum_{x \in X} x=\frac{1}{\# Y} \sum_{y \in Y} y \text { and } \# X \prod_{x \in X} x \leqslant \# Y \prod_{y \in Y} y\right) \text {. }
$$

Hereinafter, we will use the partial order $\leqslant_{\mathbb{H}}$, performing the comparison by selecting the greatest values of each THFE. Moreover, the notation $X \sim_{\mathbb{H}} Y$ will be used to express that $X$ and $Y$ are not comparable w.r.t. the order $\leqslant_{\mathbb{H}}$, i.e. neither $X \leqslant_{\mathbb{H}} Y$ nor $Y \leqslant{ }_{\mathbb{H}} X$.

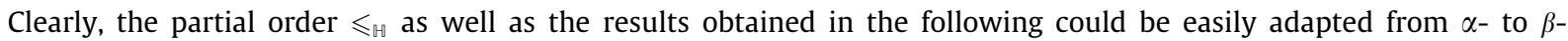
normalization.

Proposition 1. $\left(\mathbb{H}, \leqslant_{\mathbb{H}}\right)$ is a bounded lattice with the bottom and top elements given by $\mathbf{0}=\{0\}$ and $\mathbf{1}=\{1\}$, respectively.

Proof. Let $X, Y \in \mathbb{H}, m=\# X$ and $n=\# Y$. Then, the join-operation of $X$ and $Y$, denoted by $X \vee Y$, is defined as:

$$
X \vee Y= \begin{cases}\alpha(X, n), & \text { if } \alpha(X, n)=\alpha(Y, m) ; \\ \left\{\sigma_{X}(i) \vee \sigma_{\alpha(Y, m)}(i): i \in \mathbb{N}_{m}\right\} \cup\left\{\sigma_{Y}(i): i \in \mathbb{N}_{n-m}\right\}, & \text { if } \alpha(X, n) \neq \alpha(Y, m) \text { and } m \leqslant n ; \\ \left\{\sigma_{\alpha(X, n)}(i) \vee \sigma_{Y}(i): i \in \mathbb{N}_{n}\right\} \cup\left\{\sigma_{X}(i): i \in \mathbb{N}_{m-n}\right\}, & \text { otherwise. }\end{cases}
$$

And, analogously, the meet-operation of $X$ and $Y$ is given by

$$
X \wedge Y= \begin{cases}X, & \text { if } \alpha(X, n)=\alpha(Y, m) \text { and } n \leqslant m \\ Y, & \text { if } \alpha(X, n)=\alpha(Y, m) \text { and } m<n \\ \left\{\sigma_{X}(i) \wedge \sigma_{\alpha(Y, m)}(i): i \in \mathbb{N}_{m}\right\}, & \text { if } \alpha(X, n) \neq \alpha(Y, m) \text { and } m \leqslant n \\ \left\{\sigma_{\alpha(X, n)}(i) \wedge \sigma_{Y}(i): i \in \mathbb{N}_{n}\right\}, & \text { otherwise. }\end{cases}
$$

\footnotetext{
${ }^{1}$ Note that $\prec$ is not a partial order because is not antisymmetric, e.g. $s(X)=s(\{0.2,0.7,0.8\})=s(\{0.3,0.4,0.5,0.6,0.7,0.9\})$.
} 
Notice that $X \vee \mathbf{1}=\mathbf{1}$ and $X \wedge \mathbf{0}=\mathbf{0}$, and consequently $\left(\mathbb{H}, \leqslant_{H}\right)$ is a bounded lattice.

\section{Typical hesitant aggregation functions}

We consider in this section two distinct methods for extending AFs to THAFs in accordance with the Lattice Theory. In order to illustrate our proposal we focus on four classes of THAFs, generated from the AFs in Section 2. We study the conditions under which the idempotency and monotonicity properties, the annihilator and neutral elements together with the divisors of zero are verified by $n$-ary THAFs. Additionally other properties are also investigated for binary THAFs.

\subsection{Main concepts of THAFs}

We present in this section two methods to generate THAFs from AFs, both preserving monotonicity with respect to the partial order $\leqslant_{H}$ and the boundary conditions:

Definition 9. Let $\mathcal{M}: \mathbb{H}^{n} \rightarrow \mathbb{H}$ be an $n$-ary function.

(i) $\mathcal{M}$ is an $n$-ary THAF if

[M1] $\mathcal{M}$ is isotonic w.r.t. the partial order $\leqslant_{H}$ such that $\mathcal{M}(\mathbf{0}, \ldots, \mathbf{0})=\mathbf{0}$ and $\mathcal{M}(\mathbf{1}, \ldots, \mathbf{1})=\mathbf{1}$.

By Definition 9, the maximun and minimun operators are extended to HFL:

Example 4. Let $X_{1}, X_{2}, \ldots, X_{n} \in \mathbb{H}$. The operators $\mathcal{M}_{\wedge}, \mathcal{M}_{\vee}: \mathbb{\boxplus}^{n} \rightarrow \mathbb{U}$ given by

$$
\mathcal{M}_{\wedge}\left(X_{1}, \ldots, X_{n}\right)=X_{1} \wedge \ldots \wedge X_{n}, \quad \mathcal{M}_{\vee}\left(X_{1}, \ldots, X_{n}\right)=X_{1} \vee \ldots \vee X_{n}
$$

are THAFs. Moreover, any combination of $\Lambda$ and $\vee$ is also a THAF. See, e.g, the max-min functions

$$
B_{S}\left(X_{1}, \ldots, X_{n}\right)=\bigvee_{i=1 X_{j} \in S_{i}}^{m} \bigwedge_{j}
$$

where $S=\left\{S_{i}\right\}_{i=1}^{m}$ is a family of subsets of $\left\{X_{1}, \ldots, X_{n}\right\}$.

Proposition 2. Let $M:[0,1]^{n} \rightarrow[0,1]$ be an $n$-ary $A F, \sigma_{X}: \mathbb{N}_{\# X} \rightarrow X$ be a permutation and $\alpha_{\left(A_{m}\right)}: \mathbb{H} \times \mathbb{N} \rightarrow \mathbb{U}$ be an $\alpha$-normalization determined by $\left(A_{m}\right)_{m \in \mathbb{N}_{n}}$. Then, for all $X_{1}, \ldots, X_{n} \in \mathbb{U}$, an $n$-ary function $\widetilde{M}: \mathbb{U}^{n} \rightarrow \mathbb{U}$ given by

$$
\widetilde{M}\left(X_{1}, \ldots, X_{n}\right)= \begin{cases}\left\{M\left(\sigma_{X_{1}}(i), \ldots \sigma_{X_{n}}(i)\right): 1 \leqslant i \leqslant m\right\}, & \text { if } \# X_{1}=\ldots=\# X_{n} ; \\ \widetilde{M}\left(\alpha\left(X_{1}, m\right), \ldots, \alpha\left(X_{n}, m\right)\right), & \text { otherwise; }\end{cases}
$$

is a THAF when $m=\min \left\{\# X_{1}, \ldots, \# X_{n}\right\}$.

Proof. $\widetilde{M}(\mathbf{0}, \ldots, \mathbf{0})=\widetilde{M}(\{0\}, \ldots,\{0\})=\{M(0, \ldots, 0)\}=\{0\}=\mathbf{0}$. Analogously, $\widetilde{M}(\mathbf{1}, \ldots, \mathbf{1})=\mathbf{1}$. Without loss of generality, we analyze the isotonicity with respect to the first component. Let $X_{1}, \ldots, X_{n}, Y_{1}, \ldots, Y_{n} \in \mathbb{H}$. If $X_{j}<_{\mathbb{H}} Y_{j}$ then we have that $\alpha\left(X_{j}, m\right) \leqslant_{\left.\mathbb{H}^{(}\right)} \alpha\left(Y_{j}, m\right)$, for all $1 \leqslant j \leqslant n$. For simplicity, we will denote $\alpha\left(X_{j}, m\right)$ by $\widetilde{X_{j}}$. Thus, since $M$ is a non-decreasing function, for each $1 \leqslant i \leqslant m, M\left(\sigma_{\widetilde{X_{1}}}(i), \ldots, \sigma_{\widetilde{X_{n}}}(i)\right) \leqslant M\left(\sigma_{\widetilde{Y}_{1}}(i), \ldots, \sigma_{\widetilde{Y}_{n}}(i)\right)$. Therefore, we obtain that $\widetilde{M}\left(\widetilde{X_{1}}, \ldots, \widetilde{X_{n}}\right) \leqslant \widetilde{M}\left(\widetilde{Y_{1}}, \ldots, \widetilde{Y_{n}}\right)$. So, we can conclude that $\widetilde{M}\left(X_{1}, \ldots, X_{n}\right) \leqslant{ }_{H} \widetilde{M}\left(Y_{1}, \ldots, Y_{n}\right)$.

Another natural and simple way to obtain THAFs from an AFs is the following:

Proposition 3. Let $M:[0,1]^{n} \rightarrow[0$,$] be an AF. Then, for all X_{1}, \ldots, X_{n} \in \mathbb{U}$, a function $\widehat{M}: \mathbb{\boxplus}^{n} \rightarrow \mathbb{U}$ given by Eq. (13), is a THAF.

$$
\widehat{M}\left(X_{1}, X_{2}, \ldots, X_{n}\right)=\left\{M\left(x_{1}, \ldots, x_{n}\right): x_{i} \in X_{i} \text { for each } i=1, \ldots, n\right\}
$$

Proof. $\widehat{M}(\mathbf{0}, \ldots, \mathbf{0})=\widetilde{M}(\{0\}, \ldots,\{0\})=\{M(0, \ldots, 0)\}=\{0\}=\mathbf{0}$. Analogously, $\widehat{M}(\mathbf{1}, \ldots, \mathbf{1})=\mathbf{1}$. Let $X_{1}, Y_{1} \in \mathbb{U}$. Again, we just analyze the isotonicity with respect to the first component. First, suppose that $\# Y_{1} \leqslant \# X_{1}$ then, for each $y_{1} \in Y_{1}$ and $x_{i} \in X_{i}$ such that $2 \leqslant i \leqslant n$, it holds that $M\left(\sigma_{X_{1}}\left(\sigma_{Y_{1}}^{-1}\left(y_{1}\right)\right), x_{2}, \ldots, x_{n}\right) \leqslant M\left(y_{1}, x_{2}, \ldots, x_{n}\right)$. So, clearly, $\widehat{M}\left(X_{1}, X_{2}, \ldots, X_{n}\right)$ $\leqslant \widehat{M}\left(Y_{1}, X_{2}, \ldots, X_{n}\right)$. And now, in other case, if \# $Y_{1}<\# X_{1}$ then, for each $x_{i} \in X_{i}$ such that $1 \leqslant i \leqslant n$, we have that $M\left(x_{1}, \ldots, x_{n}\right) \leqslant M\left(\sigma_{Y_{1}}\left(\sigma_{X_{1}}^{-1}\left(x_{1}\right)\right), \ldots, x_{n}\right)$. Therefore, $\widehat{M}\left(X_{1}, X_{2}, \ldots, X_{n}\right) \leqslant{ }_{H} \widehat{M}\left(Y_{1}, X_{2}, \ldots, X_{n}\right)$.

In Remark 2, we briefly recall some well-known HAFs, in the sense of [38]. 
Table 1

Examples of THAFs.

\begin{tabular}{ll}
\hline k Operator & THAFs \\
\hline \multirow{3}{*}{ 1 HFWA } & $\mathbb{M}_{1}\left(X_{1}, X_{2}\right)=\{0.72,0.54 .0 .6,0.42,0.8,0.62\}$ \\
& $\widehat{M_{1}}\left(X_{1}, X_{2}\right)=\{0.8,0.54\}$ \\
& $\widehat{M_{1}}\left(X_{1}, X_{2}\right)=\{0.72,0.54,0.8,0.62\}$ \\
& $\mathbb{M}_{2}\left(X_{1}, X_{2}\right)=\{0.2904,0.2724,0.348,0.33,0.48,0.462\}$ \\
3 HFOWA & $\widehat{M_{2}}\left(X_{1}, X_{2}\right)=\{0.512,0.87\}$ \\
& $\widehat{M_{2}}\left(X_{1}, X_{2}\right)=\{0.888,0.87,0.512,0.694\}$ \\
& $\mathbb{M}_{3}\left(X_{1}, X_{2}\right)=\{0.68,0.56,0.5,0.38,0.8,0.68\}$ \\
& $\widehat{M_{3}}\left(X_{1}, X_{2}\right)=\widehat{M_{1}}\left(X_{1}, X_{2}\right)$ \\
& $\widehat{M_{3}}\left(X_{1}, X_{2}\right)=\widehat{M_{1}}\left(X_{1}, X_{2}\right)$ \\
& $\mathbb{M}_{4}\left(X_{1}, X_{2}\right)=\{0.228,0.216,0.282,0.27,0.48,0.468\}$ \\
& $\widehat{M_{4}}\left(X_{1}, X_{2}\right)=\widehat{M_{2}}\left(X_{1}, X_{2}\right)$ \\
& $\widehat{M_{4}}\left(X_{1}, X_{2}\right)=\widehat{M_{2}}\left(X_{1}, X_{2}\right)$ \\
\hline
\end{tabular}

Remark 2. Let $\left(w_{1}, w_{2}, \ldots, w_{n}\right) \in[0,1]^{n}$ be a non-negative weighting vector such that $\sum_{i=1}^{n} w_{i}=1$. Additionally, for all $\left(X_{1}, X_{2}, \ldots, X_{n}\right) \in \mathbb{U}^{n}$, let $\sigma$ be a permutation ordering such elements in a way that $X_{\sigma(i)}$ is the $i$-th largest element of all $X_{i}$ and whenever $i=1(1) n$ and $0 \leqslant l_{i} \leqslant 1, l_{i}$ are the corresponding confident levels of $X_{i}$. For all $\left(X_{1}, X_{2}, \ldots, X_{n}\right) \in \mathbb{U}^{n}$ we report the following definitions:

1. Hesitant Fuzzy Weighted Averaging Operator, HFWA : $\mathbb{\sharp}^{n} \rightarrow \mathbb{\sharp}$ given by

$$
\operatorname{HFWA}\left(X_{1}, X_{2}, \ldots, X_{n}\right)=\bigcup_{x_{1} \in X_{1}, \ldots, x_{n} \in X_{n}} \sum_{i=1}^{n} w_{i} \cdot x_{i}
$$

2. Hesitant Fuzzy Confidence Induced Weighted Aggregation Operator, denoted by CIHFWA $: \mathbb{\boxplus}^{n} \rightarrow \mathbb{U}$ and given by

$$
\operatorname{CIHFWA}\left(X_{1}, X_{2}, \ldots, X_{n}\right)=\bigcup_{x_{1} \in X_{1}, \ldots, x_{n} \in X_{n}} \sum_{i=1}^{n} w_{i} \cdot l_{i} \cdot x_{i}
$$

3. Hesitant Fuzzy Ordered Weighted Averaging Operator, HFOWA : $\mathbb{U}^{n} \rightarrow \mathbb{U}$ given by

$$
\operatorname{HFOWA}\left(X_{1}, X_{2}, \ldots, X_{n}\right)=\bigcup_{x_{1} \in X_{1}, \ldots, x_{n} \in X_{n}} \sum_{i=1}^{n} w_{i} \cdot x_{\sigma(i)}
$$

4. Confidence Induced Ordered Weighted Aggregation Operator, CIHFOWA $: \mathbb{\boxplus}^{n} \rightarrow \llbracket$ given by

$$
\operatorname{CIHFOWA}\left(X_{1}, X_{2}, \ldots, X_{n}\right)=\bigcup_{x_{1} \in X_{1}, \ldots, X_{n} \in X_{n}} \sum_{i=1}^{n} w_{i} \cdot l_{\sigma(i)} \cdot x_{\sigma(i)} .
$$

Table 1 presents three examples for the above four classes of THAFs which are conceived with three distinct definitions. The first one, indexed by $\mathbb{M}_{k}, k \in \mathbb{N}_{4}$, is related to the operators reported in Remark 2; the second and third definitions are related to the methodologies to obtain THFSs, given by Definitions 2 and 3, respectively. In Table 1 we consider the following THFEs,

$$
X_{1}=\{0.6,0.3,0.8\}, \quad X_{2}=\{0.8 .0 .5\} \in \mathbb{U}
$$

as well as the weighting vector $w=(0.4,0.6)$. The examples of CIHFWAs and CIHFOWAs are obtained when we consider that, for $i \in \mathbb{N}_{2}$ and $j \in \mathbb{N}_{3}$, each $x_{i j} \in X_{i}$ is associated to a confidence level $l_{i j}$. In this case-study, they are expressed as $\left.X_{1}^{l}=\{(0.1,0.6),(0.5,0.3),(0.6,0.8)\}\right)$ and $X_{2}^{l}=\{(0.6,0.8),(0.9,0.5)\}$.

Some comments related to the THAFs in Table 1 :

(i) $\mathbb{M}_{k}$, with $k \in \mathbb{N}_{4}$, were obtained by applying Eqs. (14)-(17) making explicit all the information represented by the THFSs;

(ii) Since $\alpha\left(X_{1}, 2\right) \leqslant{ }_{H^{2}} X_{2}$, by Eq. (9) we have that $X_{1} \geqslant_{H_{H}} X_{2}$. This implies that:

(ii-a) By Eq. (12), it holds that

$$
\widetilde{M_{1}}\left(X_{1}, X_{2}\right)=\widetilde{M_{1}}\left(\{0.6,0.8\}, X_{2}\right)=\{0.8 \cdot 0.4+0.8 \cdot 0.6,0.6 \cdot 0.4+0.5 \cdot 0.6\},
$$

resulting on the simplified expression of $\widetilde{M_{1}}$ in Table 1 . One can observe that when $M$ is an AF, only the relevant information is extracted from both the partial order $\leqslant_{\mathbb{H}}$ and the $\alpha$-normalization process is represented by the THAF $\widetilde{M}$. 
(ii-b) Additionally, by Eq. (13), it holds that

$$
\begin{aligned}
\widehat{M}_{1}\left(X_{1}, X_{2}\right)= & \widehat{M}_{1}\left(\{0.6,0.8\}, X_{2}\right) \\
= & \{0.6 \cdot 0.4+0.8 \cdot 0.6,0.6 \cdot 0.4+0.5 \cdot 0.6,0.3 \cdot 0.4+0.8 \cdot 0.6,0.3 \cdot 0.4+0.5 \cdot 0.6,0.8 \cdot 0.4+0.8 \cdot 0.6,0.8 \\
& \cdot 0.4+0.5 \cdot 0.6\} .
\end{aligned}
$$

See line 3 at Table 1 . Now, when $M$ is an AF, the relevant information, which is extracted from $\alpha$-normalization process is represented by the THAF $\widehat{M}$.

(iii) According to Eqs. (12) and (13) together with the statements in Example 3, since $X_{2} \leqslant_{\mathbb{H}} X_{1}$, we have that

$$
\widetilde{M_{3}}\left(X_{1}, X_{2}\right)=\widetilde{M_{1}}\left(X_{1}, X_{2}\right)
$$

and $\widehat{M_{3}}\left(X_{1}, X_{2}\right)=\widehat{M_{1}}\left(X_{1}, X_{2}\right)$, as stated in Table 1 . Analogous considerations can be obtained based on the fact that $X_{1}^{l} \geqslant{ }_{\mathbb{M}}^{l}$.

(iv) By Remark $1, X_{1} \prec X_{2}$. Thus, by applying Eq. (16), we obtain that

$$
\begin{aligned}
M_{3}= & \{0.8 \cdot 0.4+0.6 \cdot 0.6,0.8 \cdot 0.4+0.8 \cdot 0.6,0.8 \cdot 0.4+0.3 \cdot 0.6,0.5 \cdot 0.4+0.6 \cdot 0.6,0.5 \cdot 0.4+0.8 \cdot 0.6,0.5 \cdot 0.4 \\
& +0.3 \cdot 0.6\} .
\end{aligned}
$$

Analogously, since $X_{1}^{l} \prec X_{2}^{l}$, it results in

$$
\begin{aligned}
\mathbb{M}_{4}= & \{(0.6 \cdot 0.8) \cdot 0.4+(0.1 \cdot 0.6) \cdot 0.6,(0.6 \cdot 0.8) \cdot 0.4+(0.5 \cdot 0.3) \cdot 0.6,(0.6 \cdot 0.8) \cdot 0.4+(0.6 \cdot 0.8) \cdot 0.6,(0.9 \cdot 0.5) \\
& \cdot 0.4+(0.1 \cdot 0.6) \cdot 0.6,(0.9 \cdot 0.5) \cdot 0.4+(0.5 \cdot 0.3) \cdot 0.6,(0.9 \cdot 0.5) \cdot 0.4+(0.6 \cdot 0.8) \cdot 0.6\}
\end{aligned}
$$

Both simplified results are included in Table 1.

\subsection{Properties of AFs preserved by THAFs}

Firstly, this section reports the main properties of AFs based on remarkable works [10,11,17-19,27,43]. After this conceptual section and compatible with the two methodologies to obtain THAFs introduced in Section 4.1, by Definitions 2 and 3 , this work introduces two theorems discussing the conditions under which properties verified by AFs are preserved by their corresponding THAFs.

Definition 10. Let $\mathcal{M}: \mathbb{\boxplus}^{n} \rightarrow \mathbb{U}$ be an $n$-ary THAF.

(i) $\mathcal{M}$ has an annihilator element $A \in \mathbb{U}$ if

[M2] $\mathcal{M}\left(X_{1}, \ldots, X_{n}\right)=A$ whenever $A \in\left\{X_{1}, \ldots, X_{n}\right\}$;

(ii) $\mathcal{M}$ has a neutral element $E \in \mathbb{W}$ if

[M3] $\mathcal{M}(\underbrace{E, \ldots, E}_{(k-1) \text {-times }}, X, \underbrace{E, \ldots, E}_{(n-k) \text {-times }})=X$ for each $X \in \mathbb{H}$ and $1 \leqslant k \leqslant n$.

(iii) $\mathcal{M}$ has a divisor of zero if

[M4] there exists $\left(X_{1}, \ldots, X_{n}\right) \in(\mathbb{H} \backslash\{\mathbf{0}\})^{n}$ such that $\mathcal{M}\left(X_{1}, \ldots, X_{n}\right)=\mathbf{0}$.

(iv) $\mathcal{M}$ is idempotent if

[M5] $\mathcal{M}(X, \ldots, X)=X$ for any $X \in \mathbb{H}$.

(v-a) $\mathcal{M}$ has only trivial idempotent elements if

[M5-b] $\mathcal{M}(X, \ldots, X)=X$ iff $X=\mathbf{1}$ or $X=\mathbf{0}$.

(v) $\mathcal{M}$ is strictly isotonic if

[M6] for each $X_{1}, Y_{1}, \ldots, X_{n}, Y_{n} \in \mathbb{U} \backslash\{\mathbf{0}, \mathbf{1}\}$ such that $X_{i} \leqslant{ }_{H} Y_{i}$, for each $i=1, \ldots, n$ and $X_{j} \neq Y_{j}$ for some $j=1, \ldots, n$, $\mathcal{M}\left(X_{1}, \ldots, X_{n}\right)<_{\mathbb{H}} \mathcal{M}\left(Y_{1}, \ldots, Y_{n}\right)$.

In the following some additional definitions are considered for binary THAFs. For that, let $\mathcal{M}^{0}(X)=X$ and $\mathcal{M}^{k+1}(X)=\mathcal{M}\left(X, \mathcal{M}^{k}(X)\right)$ be the inductive definition of the binary operation.

Definition 11. Let $\mathcal{M}$ be a binary THAF. It follows that:

(vii) $\mathcal{M}$ has a nilpotent element $T \in \mathbb{U} \backslash\{\mathbf{0}, \mathbf{1}\}$ if

[M7] there exists $n \in \mathbb{N}$, such that $\mathcal{M}^{n}(T, \ldots, T)=\mathbf{0}$;

(viii) $\mathcal{M}$ is symmetric if

[M8] $\mathcal{M}\left(X_{1}, X_{2}\right)=\mathcal{M}\left(X_{2}, X_{1}\right)$, for all $X_{1}, X_{2} \in \mathbb{H}$;

(ix) $\mathcal{M}$ is associative if

[M9] $\mathcal{M}\left(\mathcal{M}\left(X_{1}, X_{2}\right), X_{3}\right)=\mathcal{M}\left(X_{1}, \mathcal{M}\left(X_{2}, X_{3}\right)\right)$ for all $X_{1}, X_{2}, X_{3} \in \mathbb{U}$; 
(x) $\mathcal{M}$ verifies the Archimidean property if

[M10] for all $X, Y \in \mathbb{U} /\{\mathbf{0}, \mathbf{1}\}$, there exists $n \in \mathbb{N}$ such that $\mathcal{M}^{n}(X)<_{\mathbb{H}} Y$;

(xi) $\mathcal{M}$ satisfies the cancelation law if

[M11] $\mathcal{M}\left(X_{1}, X_{2}\right)=\mathcal{M}\left(X_{1}, Z\right)$ implies that $X_{1}=\mathbf{0}$ or $X_{2}=Z$.

Theorem 2. Let $M:[0,1]^{n} \rightarrow[0,1]$ be a $n$-ary $A F$. Then, it follows that:

(i) $\widetilde{M}$ is an n-ary THAF iff $M$ is an n-ary aggregation function;

(ii) $\widetilde{M}$ has an annihilator element iff $M$ has an annihilator element;

(iii) $\widetilde{M}$ has a divisor of zero iff $M$ has a divisor of zero;

(iv) $\widetilde{M}$ is idempotent iff $M$ is idempotent;

(v) $\widetilde{M}$ has a nilpotent element iff $M$ has a nilpotent element;

(vi) If $n=2$ then $\widetilde{M}$ is symmetric iff $\mathcal{M}$ is symmetric;

(vii) If $n=2$ then $\widetilde{M}$ is associative iff $M$ is associative;

(viii) If $n=2$ then $\widetilde{M}$ is Archimedean iff $M$ is Archimedean;

$$
\left\{M\left(x_{1}, \ldots, x_{n}\right)\right\} \leqslant_{\mathbb{H}}\left\{M\left(y_{1}, \ldots, y_{n}\right)\right\} .
$$

Proof. Consider $X_{1}, X_{2}, \ldots, X_{n} \in \mathbb{H}$.

(i) $(\Rightarrow)$ By Definition 2, it follows that $\{M(0, \ldots, 0)\}=\widetilde{M}(\{0\}, \ldots,\{0\})=\widetilde{M}(\mathbf{0}, \ldots, \mathbf{0})=\mathbf{0}=\{0\}$. Therefore, $M(0, \ldots, 0)=0$. Analogously, $M(1, \ldots, 1)=1$. If $x_{i} \leqslant y_{i}$ for each $i \in \mathbb{N}_{n}$, then $\widetilde{M}\left(\left\{x_{1}\right\}, \ldots,\left\{x_{n}\right\}\right) \leqslant \widetilde{M}\left(\left\{y_{1}\right\}, \ldots,\left\{y_{n}\right\}\right)$ and so, by definition of $\leqslant{ }_{\mathbb{H}}, M\left(x_{1}, \ldots, x_{n}\right) \leqslant M\left(y_{1}, \ldots, y_{n}\right)$.

$(\Leftarrow)$ Straightforward from Proposition 2 .

(ii) $(\Rightarrow)$ Let $A$ be an annihilator element of $\widetilde{M}$ and $\{a\}=\alpha(A, 1)$. If $x_{i} \in[0,1]$ for any $i \in \mathbb{N}_{n}$ and $x_{k}=a$ for some $k \in \mathbb{N}_{n}$, then

$$
\begin{aligned}
A & =\tilde{M}\left(\left\{x_{1}\right\}, \ldots,\left\{x_{k-1}\right\}, A,\left\{x_{k+1}\right\}, \ldots,\left\{x_{n}\right\}\right)=\tilde{M}\left(\left\{x_{1}\right\}, \ldots,\left\{x_{k-1}\right\},\{a\},\left\{x_{k+1}\right\}, \ldots,\left\{x_{n}\right\}\right) \\
& =\left\{M\left(x_{1}, \ldots, x_{k-1}, a, x_{k+1}, \ldots, x_{n}\right)\right\}
\end{aligned}
$$

It is only possible if $\# A=1$ and, therefore $M\left(x_{1}, \ldots, x_{k-1}, a, x_{k+1}, \ldots, x_{n}\right)=a$.

$(\Leftarrow)$ Let $A=\{a\} \in\left\{X_{1}, \ldots, X_{n}\right\}$ such that $a$ is an annihilator of $M$. Then, whenever $\left\{x_{i}\right\}=\alpha\left(X_{i}, 1\right)$, for all $1 \leqslant i \leqslant n$, it holds that $\widetilde{M}\left(X_{1}, \ldots, X_{n}\right)=\widetilde{M}\left(\alpha\left(X_{1}, 1\right), \ldots, A, \ldots, \alpha\left(X_{n}, 1\right)\right)=\left\{M\left(x_{1}, \ldots, a, \ldots, x_{n}\right)\right\}=\{a\}=A$.

(iii) $(\Rightarrow)$ If $\left(X_{1}, \ldots, X_{n}\right) \in(\mathbb{U} \backslash\{\mathbf{0}\})^{n}$ is a divisor of zero of $\widetilde{M}$ and $m=\min \left(\# X_{1}, \ldots\right.$,\#跓), then $\mathbf{0}=\widetilde{M}\left(X_{1}, \ldots, X_{n}\right)=$ $\widetilde{M}\left(\widetilde{X_{1}}, \ldots, \widetilde{X_{n}}\right)=\left\{M\left(\sigma_{\widetilde{X_{1}}}(i), \ldots, \sigma_{\widetilde{X}_{n}}(i)\right): 1 \leqslant i \leqslant m\right\}$. Therefore, for each $\in \mathbb{N}_{m}, M\left(\sigma_{\widetilde{X}_{1}}(i), \ldots, \sigma_{\widetilde{X}_{n}}(i)\right)=0$, which means that $\left(\sigma_{\tilde{X}_{1}}(i), \ldots, \sigma_{\widetilde{X}_{n}}(i)\right)$ is a divisor of zero of $M$.

$(\Leftarrow)$ If $\left.\left(x_{1}, \ldots, x_{n}\right) \in\right] 0,1\left[^{n}\right.$ is a divisor of zero of $M$, then $\widetilde{M}\left(\left\{x_{1}\right\}, \ldots,\left\{x_{n}\right\}\right)=\left\{M\left(x_{1}, \ldots, x_{n}\right)\right\}=\{0\}=\mathbf{0}$. So, $\left(\left\{x_{1}\right\}, \ldots,\left\{x_{n}\right\}\right)$ is a divisor of zero of $\widetilde{M}$.

(iv) $(\Rightarrow)$ Let $\widetilde{M}$ be an idempotent function. Then for any $x \in[0,1],\{x\}=\widetilde{M}(\{x\}, \ldots,\{x\})=\{M(x, \ldots, x)\}$. Therefore, $M(x, \ldots, x)=x$.

$(\Leftarrow)$ Let $M$ be an idempotent function. It follows that $\widetilde{M}(X, \ldots, X)=\left\{M\left(\sigma_{X}(i), \ldots \sigma_{X}(i)\right): i \in \mathbb{N}_{\# X}\right\}=$ $\left.\left\{\sigma_{X}(i): i \in \mathbb{N}_{\# X}\right\}\right\}=X$.

(v) $(\Rightarrow)$ If $\widetilde{M}$ has a nilpotent element $T \in \mathbb{U} /\{\mathbf{0}, \mathbf{1}\}$, then we have that:

$$
\left\{M^{n}\left(t_{i}, \ldots, t_{i}\right): i \in \mathbb{N}_{m}\right\}=\widetilde{M}^{n}(T, \ldots, T)=\mathbf{0}=\{0\}
$$

where $t_{i}=\sigma_{T}(i)$ and $m=\overline{\bar{T}}$. Therefore, it holds that $M^{n}\left(t_{i}, \ldots, t_{i}\right)=0$ for any $i \in \mathbb{N}_{m}$, i.e. each $t_{i}$ is a nilpotent element of $M$. $(\Leftarrow)$ Suppose $M$ has a nilpotent element $t \in] 0,1\left[\right.$, then for some $n \in \mathbb{N}$ we have that: $\mathbf{0}=\left\{M^{n}(t, \ldots, t)\right\}=\widetilde{M}^{n}(\{t\}, \ldots,\{t\})$. So, $\widetilde{M}$ has $\{t\} \in \mathbb{U} \backslash\{\mathbf{0}, \mathbf{1}\}$ as a nilpotent element.

(vi) $(\Rightarrow)$ Consider that $\widetilde{M}$ verifies the symmetry property. Then, it holds that $\left\{M\left(x_{1}, x_{2}\right)\right\}=\widetilde{M}\left(\left\{x_{1}\right\},\left\{x_{2}\right\}\right)=$ $\widetilde{M}\left(\left\{x_{2}\right\},\left\{x_{1}\right\}\right)=\left\{M\left(x_{2}, x_{1}\right)\right\}$.

$(\Leftarrow)$ Now, suppose that $M$ is a symmetric function and consider $m=\min \left(\# X_{1}, \# X_{2}\right)$, then we have that:

$$
\widetilde{M}\left(X_{1}, X_{2}\right)=\widetilde{M}\left(\widetilde{X}_{1}, \widetilde{X}_{2}\right)=\left\{M\left(\sigma_{\widetilde{X}_{1}}(i), \sigma_{\widetilde{X}_{2}}(i)\right): i \in \mathbb{N}_{m}\right\}=\left\{M\left(\sigma_{\widetilde{X}_{2}}(i), \sigma_{\widetilde{X}_{1}}(i)\right): i \in \mathbb{N}_{m}\right\}=\widetilde{M}\left(X_{2}, X_{1}\right) .
$$

(vii) $\left(\Rightarrow\right.$ ) Now, if $\widetilde{M}$ is associative, then for all $x_{1}, x_{2}, x_{3} \in[0,1]$, we have that:

$$
\begin{aligned}
\left\{M\left(x_{1}, M\left(x_{2}, x_{3}\right)\right)\right\} & =\widetilde{M}\left(\alpha\left(\left\{x_{1}\right\}, 1\right), \tilde{M}\left(\alpha\left(\left\{x_{2}\right\}, 1\right), \alpha\left(\left\{x_{3}\right\}, 1\right)\right)=\tilde{M}\left(\tilde{M}\left(\alpha\left(\left\{x_{1}\right\}, 1\right), \alpha\left(\left\{x_{2}\right\}, 1\right)\right), \alpha\left(\left\{x_{3}\right\}, 1\right)\right)\right. \\
& =\left\{M\left(M\left(x_{1}, x_{2}\right), x_{3}\right)\right\}
\end{aligned}
$$


Therefore, it holds that $M\left(x_{1}, M\left(x_{2}, x_{3}\right)\right)=M\left(M\left(x_{1}, x_{2}\right), x_{3}\right)$.

$(\Leftarrow)$ Consider $X_{1}, X_{2}, X_{3} \in \mathbb{U}, h=\min \left(\# X_{1}, \# X_{2}\right), k=\min \left(\# X_{2}, \# X_{3}\right)$ and $m=\min \left(\# X_{1}, \# X_{2}, \# X_{3}\right)$.

Suppose $M$ as an associative function, then we have that:

$$
\begin{aligned}
\widetilde{M}\left(X_{1}, \widetilde{M}\left(X_{2}, X_{3}\right)\right) & =\widetilde{M}\left(X_{1}, \widetilde{M}\left(\alpha\left(X_{2}, k\right), \alpha\left(X_{3}, k\right)\right)\right)=\widetilde{M}\left(\alpha\left(X_{1}, m\right), \alpha\left(\widetilde{M}\left(\alpha\left(X_{2}, k\right), \alpha\left(X_{3}, k\right)\right), m\right)\right) \\
& =\left\{M\left(\sigma_{\widetilde{X}_{1}}(i), M\left(\sigma_{\widetilde{X}_{2}}(i), \sigma_{\widetilde{X}_{3}}(i)\right): i=1, \ldots, m\right\}=\left\{M\left(M\left(\sigma_{\widetilde{X}_{1}}(i), \sigma_{\widetilde{X}_{2}}(i)\right), \sigma_{\widetilde{X}_{3}}(i)\right): i=1, \ldots, m\right\}\right. \\
& =\widetilde{M}\left(\alpha\left(\widetilde{M}\left(\alpha\left(X_{1}, h\right), \alpha\left(X_{2}, h\right)\right), m\right), \alpha\left(X_{3}, m\right)\right)=\widetilde{M}\left(\widetilde{M}\left(\alpha\left(X_{1}, h\right), \alpha\left(X_{2}, h\right)\right), X_{3}\right)=\widetilde{M}\left(\widetilde{M}\left(X_{1}, X_{2}\right), X_{3}\right)
\end{aligned}
$$

(viii) $(\Rightarrow)$ Let $X, Y \in \mathbb{U} \backslash\{\mathbf{0}, \mathbf{1}\}$, then for each $x \in X$ and $y \in Y$, there exists $n \in \mathbb{N}$ such that $M^{n}(x)<y$. Let $n_{0}$ be the greatest of such $n$. Then, for each $x \in X$ and $y \in Y, M^{n_{0}}(x)<y$. Consequently, $\widetilde{M}^{n_{0}}(X)=\left\{M^{n_{0}}(x): x \in X\right\}<_{\mathbb{H}} Y$.

$(\Leftarrow)$ Suppose now that $M$ is Archimedean. Since $\widetilde{M}$ is Archimedean, it follows that for each $x, y \in(0,1)$, there exists $n \in \mathbb{N}$ such that $\widetilde{M}^{n}(\{x\})<_{\mathbb{H}}\{y\}$. Since $\widetilde{M}^{n}(\{x\})=\left\{M^{n}(x)\right\}$, we conclude that $M^{n}(x)<y$.

And, we conclude that Theorem 2 is verified.

Remark 3. For any aggregation function $M, \widetilde{M}$ does not have a neutral element, is not strictly isotonic and does not satisfy the cancellation law. In fact, suppose that $\widetilde{M}$ have a neutral element $E$, and let $m=\# E$, the for any $X \in \mathbb{E}^{(m)}$, we have that $X=\widetilde{M}(E, \ldots, E, X, E \ldots, E)=\widetilde{M}(E, \ldots, E, Y, E \ldots, E)=Y$, for $Y=X \cup\left\{\sum_{x \in X} x\right\}$, which is impossible. Moreover, since $\widetilde{M}(\{0.2\},\{0.3\})=\widetilde{M}(\{0.2\},\{0.3,0.4\})$ then $\widetilde{M}$ neither satisfies the cancellation law nor is strictly isotone.

Theorem 3. Let $\widehat{M}:[0,1]^{n} \rightarrow[0,1]$ be an n-ary aggregation function as in Eq. (13). Then, it follows that:

(i) $\widehat{M}$ is an n-ary THAF iff $M$ is a n-ary aggregation function;

(ii) $\widehat{M}$ has an annihilator element iff $M$ has an annihilator element;

(iii) $\widehat{M}$ has a neutral element if $M$ has a neutral element;

(iv) $\widehat{M}$ is has a divisor of zero iff $M$ has a divisor of zero;

(v) if $\widehat{M}$ is idempotent then $M$ is idempotent;

(vi) $\widehat{M}$ is strictly isotone iff $M$ is strictly increasing;

(vii) $\widehat{M}$ has a nilpotent element iff $M$ has a nilpotent element;

(viii) If $n=2$ then $\widehat{M}$ is symmetric iff $\mathcal{M}$ is symmetric;

(ix) If $n=2$ then $\widehat{M}$ is associative iff $M$ is associative;

(x) If $n=2$ then $\widehat{M}$ is Archimedean then $M$ is Archimedean;

(xi) If $n=2$ and $\widehat{M}$ satisfies the cancelation law then M satisfies the cancelation law.

(xii) If $n=2, M$ is strict and satisfies the cancellation law, then $\widehat{M}$ satisfies the cancelation law.

\section{Proof}

(i) $(\Rightarrow)$ By Definition 2, it follows that $\widehat{M}(\mathbf{0}, \ldots, \mathbf{0})=\{M(0, \ldots, 0)\}=\{0\}=\mathbf{0}$. Analogously, $\widehat{M}(\mathbf{1}, \ldots, \mathbf{1})=\mathbf{1}$. For each $i \in \mathbb{N}_{n}$, if $x_{i} \leqslant y_{i}$ then $\left\{x_{i}\right\} \leqslant_{H}\left\{y_{i}\right\}$. So, $\left\{M\left(x_{1}, \ldots, x_{n}\right)\right\}=\widehat{M}\left(\left\{x_{1}\right\}, \ldots,\left\{x_{n}\right\}\right) \leqslant{ }_{H} \widehat{M}\left(\left\{y_{1}\right\}, \ldots,\left\{y_{n}\right\}\right)=\left\{M\left(y_{1}, \ldots, y_{n}\right)\right\}$. Therefore, $M\left(x_{1}, \ldots, x_{n}\right) \leqslant M\left(y_{1}, \ldots, y_{n}\right)$.

$(\Leftrightarrow)$ Straightforward from Proposition 3 .

(ii) $(\Rightarrow)$ Let $A$ be an annihilator of $\widehat{M}, x_{i} \in[0,1]$ for any $i=1, \ldots, n$. Then for every $k \in \mathbb{N}_{n}$ we have that

$A=\widehat{M}\left(\left\{x_{1}\right\}, \ldots,\left\{x_{k-1}\right\}, A,\left\{x_{k+1}\right\}, \ldots,\left\{x_{n}\right\}\right)=\left\{M\left(x_{1}, \ldots, x_{k-1}, a, x_{k+1}, \ldots, x_{n}\right): a \in A\right\}$

So, for each $a \in A$, it follows that $M\left(x_{1}, \ldots, x_{k-1}, a, x_{k+1}, \ldots, x_{n}\right)=a$.

$(\Leftarrow)$ Let $A=\{a\} \in\left\{X_{1}, \ldots, X_{n}\right\}$ such that $a$ is an annihilator of $M$. Then, for all $1 \leqslant i \leqslant n$, it holds that $\widehat{M}\left(X_{1}, \ldots, A, \ldots, X_{n}\right)=\left\{M\left(x_{1}, \ldots, a, \ldots, x_{n}\right): x_{i} \in X_{i}\right\}=\{a\}=A$.

(iii) Let $e$ be the neutral element of $M$. Then it holds that:

$$
\widehat{M}(\underbrace{\{e\}, \ldots,\{e\}}_{(k-1) \text {-times }}, X,\{\underbrace{\{e\}, \ldots,\{e\}}_{(n-k) \text {-times }})=\{M(\underbrace{e, \ldots, e}_{(k-1) \text {-times }} x \underbrace{e, \ldots, e}_{(n-k) \text {-times }}): x \in X\}=X .
$$

(iv) $(\Rightarrow)$ If $\left.\left(x_{1}, \ldots, x_{n}\right) \in\right] 0,1{ }^{n}$ is a divisor of zero of $M$, then $\widehat{M}\left(\left\{x_{1}\right\}, \ldots,\left\{x_{n}\right\}\right)=\left\{M\left(x_{1}, \ldots, x_{n}\right)\right\}=\{0\}=\mathbf{0}$. So, $\left(\left\{x_{1}\right\}, \ldots,\left\{x_{n}\right\}\right)$ is a divisor of zero of $\widetilde{M}$.

$(\Leftarrow)$ If $\left(X_{1}, \ldots, X_{n}\right) \in(\mathbb{H}\{\mathbf{0}\})^{n}$ is a divisor of zero of $\widehat{M}$ then $\{0\}=\widehat{M}\left(X_{1}, \ldots, X_{n}\right)=\left\{M\left(x_{1}, \ldots, x_{n}\right): x_{i} \in X_{i}\right.$ for each $\left.i \in \mathbb{N}_{n}\right\}$. Therefore, for every $x_{i} \in X_{i}$, with $i \in \mathbb{N}_{n}, M\left(x_{1}, \ldots, x_{n}\right)=0$.

(v) Suppose that $\widehat{M}$ is an idempotent function on $\mathbb{H}$. Then, for any $x \in[0,1],\{x\}=\widehat{M}(\{x\}, \ldots,\{x\})=\{M(x, \ldots, x)\}$. Therefore, $M(x, \ldots, x)=x$. 
(vi) $\left(\Rightarrow\right.$ ) Suppose that $\widehat{M}$ is strictly increasing function on $\mathbb{\llbracket}$. Taking $x_{i}, y_{i} \in[0,1]$ such that $x_{i} \leqslant y_{i}$ for $i \in \mathbb{N}_{n}$ and $x_{j} \neq y_{j}$ for some $j \in \mathbb{N}_{n}$, we obtain $X_{i}=\left\{x_{i}\right\} \leqslant_{\mathbb{H}}\left\{y_{i}\right\}=Y_{i}$ for each $i$ and $X_{j} \neq Y_{j}$ for some $j$, which means that: $\left\{M\left(x_{1}, \ldots, x_{n}\right)\right\}=\widehat{M}\left(X_{1}, \ldots, X_{n}\right)<_{\mathbb{H}} \widehat{M}\left(Y_{1}, \ldots, Y_{n}\right)=\left\{M\left(y_{1}, \ldots, y_{n}\right)\right\}$. Therefore, $M\left(x_{1}, \ldots, x_{n}\right)<M\left(y_{1}, \ldots, y_{n}\right)$.

$(\Leftarrow)$ Suppose that $M$ is strictly increasing. Without loss of generality, we consider the case $\widehat{M}\left(X, X_{2}, \ldots, X_{n}\right)$ and $\widehat{M}\left(Y, X_{2}, \ldots, X_{n}\right)$ for $X_{2}, \ldots, X_{n}, X, Y \in \mathbb{H}$ when $X<_{\mathbb{H}} Y$. By Eq. (9) we have the following two cases:

1. If $\alpha(X, m)<_{\mathbb{H}^{(m)}} \alpha(Y, m)$, where $m=\min (\# X, \# Y)$, then by Eq. (6), for each $1 \leqslant i \leqslant m$ we have that $\sigma_{X}(i) \leqslant \sigma_{Y}(i)$ and $\sigma_{X}(k)<\sigma_{Y}(k)$ for some $1 \leqslant k \leqslant m$. So, because $M$ is isotone, $M\left(\sigma_{X}(i), x_{2}, \ldots, x_{n}\right) \leqslant M\left(\sigma_{Y}(i), x_{2}, \ldots, x_{n}\right)$ for each $i \in \mathbb{N}_{m}$. And because $M$ is strictly isotone, $M\left(\sigma_{X}(k), x_{2}, \ldots, x_{n}\right) \leqslant M\left(\sigma_{Y}(k), x_{2}, \ldots, x_{n}\right)$ for some $k \in \mathbb{N}_{m}$. Therefore, $\widehat{M}\left(X, X_{2}, \ldots, X_{n}\right)=\left\{M\left(x, x_{2}, \ldots, x_{n}\right): x_{i} \in X_{i}\right.$ for $i=2, \ldots, n$ and $\left.x \in X\right\}<_{\mathbb{H}}\left\{M\left(y, x_{2}, \ldots, x_{n}\right): x_{i} \in X_{i}\right.$ for $i=2, \ldots, n$ and $y \in Y\}=\widehat{M}\left(Y, X_{2}, \ldots, X_{n}\right)$

2. If $\alpha(X, m)=\alpha(Y, m)$ and $\# Y<\# X$, for each $i>p=\# \widehat{M}\left(Y, X_{2}, \ldots, X_{n}\right), \sigma_{\widehat{M}\left(X, X_{2}, \ldots, X_{n}\right)}(i)$ is an upper bound of $\widehat{M}\left(\alpha(X, m), X_{2}, \ldots, X_{n}\right), \quad$ and $\quad$ so $\quad \alpha\left(\widehat{M}\left(X, X_{2}, \ldots, X_{n}\right), p\right)=\alpha\left(\widehat{M}\left(Y, X_{2}, \ldots, X_{n}\right), p\right)$. Thus, since $\# \widehat{M}\left(Y, X_{2}, \ldots, X_{n}\right)<\# \widehat{M}\left(X, X_{2}, \ldots, X_{n}\right)$, then $\widehat{M}\left(X, X_{2}, \ldots, X_{n}\right)<_{H} \widehat{M}\left(Y, X_{2}, \ldots, X_{n}\right)$.

(vii) $(\Rightarrow)$ Suppose $M$ has a nilpotent element $t \in] 0,1\left[\right.$, then for $n \in \mathbb{N}$ we have that $\widehat{M}^{n}(\{t\})=\left\{M^{n}(t)\right\}=\mathbf{0}$. So, $\widehat{M}$ has the nilpotent element $\{t\} \in \mathbb{H} \backslash\{\mathbf{0}, \mathbf{1}\}$.

$(\Leftarrow)$ If $\widehat{M}$ has a nilpotent element $T \in \mathbb{M} \backslash\{\mathbf{0}, \mathbf{1}\}$, it follows that: $\left\{M^{n}(t): t \in T\right\} \subseteq \widehat{M}^{n}(T)=\mathbf{0}$. Therefore, $M^{n}(t)=0$ for each $t \in T$.

(viii) $(\Leftarrow)$ Consider that $\widehat{M}$ verifies the symmetry property. Then, it holds that $\left\{M\left(x_{1}, x_{2}\right)\right\}=\widehat{M}\left(\left\{x_{1}\right\},\left\{x_{2}\right\}\right)=$ $\widehat{M}\left(\left\{x_{2}\right\},\left\{x_{1}\right\}\right)=\left\{M\left(x_{2}, x_{1}\right)\right\}$. So, $M$ satisfies the symmetry property.

$(\Rightarrow)$ Conversely, suppose that $M$ is a symmetric function, then we have that:

$$
\widehat{M}\left(X_{1}, X_{2}\right)=\left\{M\left(x_{1}, x_{2}\right): x_{i} \in X_{i}, i \in\{1,2\}\right\}=\left\{M\left(x_{2}, x_{1}\right): x_{i} \in X_{i}, i \in\{1,2\}\right\}=\widehat{M}\left(X_{2}, X_{1}\right) .
$$

(ix) $(\Rightarrow)$ Let $M$ be an associative function, for all $X_{1}, X_{2}, X_{3} \in[0,1]$, we have that:

$$
\begin{gathered}
\widehat{M}\left(X_{1}, \widehat{M}\left(X_{2}, X_{3}\right)\right)=\widehat{M}\left(X_{1},\left\{M\left(x_{2}, x_{3}\right): x_{i} \in X_{i}, i \in\{2,3\}\right\}\right)=\left\{M\left(x_{1}, M\left(x_{2}, x_{3}\right)\right): x_{i} \in X_{i}, i \in\{1,2,3\}\right\}=\left\{M\left(M\left(x_{1}, x_{2}\right), x_{3}\right)\right. \\
\left.\left.: x_{i} \in X_{i}, i \in\{1,2,3\}\right\}=\widehat{M}\left(\left\{M\left(x_{1}, x_{2}\right): x_{i} \in X_{i}, i \in\{1,2\}\right\}, X_{3}\right)=\widehat{M}\left(\widehat{M}\left(X_{1}, X_{2}\right), X_{3}\right)\right)
\end{gathered}
$$

$(\Leftarrow)$ Now, if $\widehat{M}$ is associative then, for all $x_{1}, x_{2}, x_{3} \in[0,1]$, we have that:

$$
\left\{M\left(x_{1}, M\left(x_{2}, x_{3}\right)\right)\right\}=\widehat{M}\left(\left\{x_{1}\right\}, \widehat{M}\left(\left\{x_{2}\right\},\left\{x_{3}\right\}\right)\right)=\widehat{M}\left(\widehat{M}\left(\left\{x_{1}\right\},\left\{x_{2}\right\}\right),\left\{x_{3}\right\}\right)=\left\{M\left(M\left(x_{1}, x_{2}\right), x_{3}\right)\right\}
$$

Therefore, it holds that $M\left(x_{1}, M\left(x_{2}, x_{3}\right)\right)=M\left(M\left(x_{1}, x_{2}\right), x_{3}\right)$.

$(x)(\Rightarrow)$ Let $X, Y \in \mathbb{U} \backslash\{\mathbf{0}, \mathbf{1}\}$, then for each $x \in X$ and $y \in Y$, there exists $n \in \mathbb{N}$ such that $M^{n}(x)<y$. Let $n_{0}$ the greater of such $n$. Then, for each $x \in X$ and $y \in Y, M^{n_{0}}(x)<y$. So, $\widehat{M}^{n_{0}}(X) \leqslant_{\mathbb{H}}\left\{M^{n_{0}}(x): x \in X\right\}<_{\mathbb{H}} Y$.

$(\Leftarrow)$ Suppose now that $M$ is Archimedean. Since $\widehat{M}$ is Archimedean, it follows that for each $x, y \in(0,1)$ there exists $n \in \mathbb{N}$ such that $\widehat{M}^{n}(\{x\})<_{\mathbb{H}}\{y\}$. So, because $\widehat{M}^{n}(\{x\})=\left\{M^{n}(x)\right\}$, we have that, $M^{n}(x)<y$.

(xi) Suppose that $\widehat{M}$ verifies the cancellation law. If $M\left(x_{1}, x_{2}\right)=M\left(x_{1}, z\right)$ then $\widehat{M}\left(\left\{x_{1}\right\},\left\{x_{2}\right\}\right)=\widehat{M}\left(\left\{x_{1}\right\},\{z\}\right)$, what implies $\left\{x_{1}\right\}=\mathbf{0}$ or $\left\{x_{2}\right\}=\{z\}$, or equivalently that $x_{1}=0$ or $x_{2}=z$, respectively. Therefore, $M$ verifies the cancellation law.

(xii) Let $M$ be a function satisfying the cancelation law. We prove by induction in $X_{1}$ that if, $\widehat{M}\left(X_{1}, X_{2}\right)=\widehat{M}\left(X_{1}, Z\right)$ then $X_{1}=\mathbf{0}$ or $X_{2}=Z$. The base case is $X_{1}=\left\{x_{1}\right\}$. In this case $\widehat{M}\left(X_{1}, X_{2}\right)=\widehat{M}\left(X_{1}, Z\right)$ means that $\left\{M\left(x_{1}, x_{2}\right): x_{2} \in X_{2}\right\}=\left\{M\left(x_{1}, z\right): Z \in Z\right\}$ and so for each $x_{2} \in X_{2}$ exists $z \in Z$ such that $M\left(x_{1}, x_{2}\right)=M\left(x_{1}, z\right)$ and for each $z \in Z$ exists $x_{2} \in X_{2}$ such that $M\left(x_{1}, x_{2}\right)=-$ $M\left(x_{1}, z\right)$. Because $M$ satisfies the cancellation law, either $x_{1}=0$ or $x_{2}=z$. Therefore, if $x_{1}=0$ then $X_{1}=\mathbf{0}$ and if $x_{1} \neq 0$ then for each $x_{2}$ exists $z \in Z$ such that $x_{2}=z$ and for each $z \in Z$ there exists $x_{2} \in X_{2}$ such that $x_{2}=z$. Therefore, in this last case, $X_{2}=Z$.

Suppose, that $x_{1} \notin X_{1}$ and $x_{1}$ is an upper bound of $X_{1}$. If $\widehat{M}\left(X_{1} \cup\left\{x_{1}\right\}, X_{2}\right)=\widehat{M}\left(X_{1} \cup\left\{x_{1}\right\}, Z\right)$, then $\widehat{M}\left(X_{1}, X_{2}\right) \cup \widehat{M}\left(\left\{x_{1}\right\}, X_{2}\right)=\widehat{M}\left(X_{1}, Z\right) \cup \widehat{M}\left(\left\{x_{1}\right\}, Z\right)$. Because $M$ is strict in the first component, then $\widehat{M}\left(X_{1}, X_{2}\right) \cap \widehat{M}\left(\left\{x_{1}\right\}, X_{2}\right)=\widehat{M}\left(X_{1}, Z\right) \cap \widehat{M}\left(\left\{x_{1}\right\}, Z\right)=\emptyset$. Therefore, $\widehat{M}\left(X_{1}, X_{2}\right)=\widehat{M}\left(X_{1}, Z\right)$ and $\widehat{M}\left(\left\{x_{1}\right\}, X_{2}\right)=\widehat{M}\left(\left\{x_{1}\right\}, Z\right)$. Thus, from the inductive hypotheses, $X_{1}=\mathbf{0}$ or $X_{2}=Z$, and by base case, $\left\{x_{1}\right\}=\mathbf{0}$ or $X_{2}=Z$. So, $X_{2}=Z$ or $X_{1}=\{0\}=\left\{x_{1}\right\}$ which means that $X_{1} \cup\left\{x_{1}\right\}=\mathbf{0}$.

And, we conclude that Theorem 3 is verified.

\subsubsection{Representable THAFs}

In the following, the embryonic idea of representation in the context of THAFs is introduced. Such idea extends the interval representation as presented in $[5-7,16,31]$ to the context of THAFs.

Definition 12. Let $\mathcal{M}$ be an $n$-ary THAF. $\mathcal{M}$ is an $\mathbb{M}$-representation of an $n$-ary $\mathrm{AF} M$, denoted by $\mathcal{M} \ll{ }_{\sharp} M$, if $M\left(x_{1}, \ldots, x_{n}\right) \in \mathcal{M}\left(X_{1}, \ldots, X_{n}\right)$ when $X_{1}, \ldots, X_{n} \in \mathbb{H}$ and $x_{i} \in X_{i}$ for any $i \in \mathbb{N}_{n}$. 


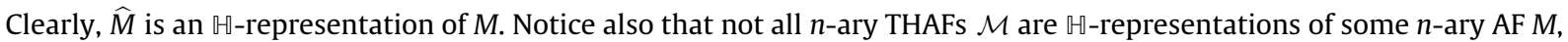
e.g., $\mathcal{M}: \mathbb{W} \rightarrow \mathbb{W}$ as a THAF defined by $\mathcal{M}(X)=\left\{\sigma_{X}(\# X)\right\}$, or equivalently, $\mathcal{M}(X)=\{\max (X)\}$, for each $X \in \mathbb{H}$.

\section{Finite hesitant triangular norms}

Extending the study of t-norm class, one of the most relevant class of aggregation functions which plays an important role in many applications of Fuzzy Logic [16,23], in this section we discuss the condition under which an $\square-t-s u b n o r m$ can be obtained from a t-subnorm by applying the two methodologies introduced in Section 4.

Definition 13. Let $\mathcal{T}$ be a binary THAF. $\mathcal{T}$ is said to be a typical hesitant t-subnorm, $\mathbb{-}$-t-subnorm in short, if it is

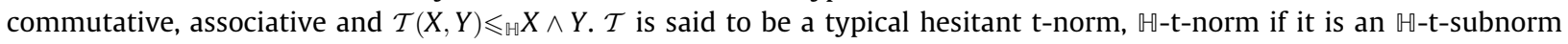
with 1 as the neutral element.

Lemma 1. Let $M_{1}$ and $M_{2}$ two AFs. $M_{1} \leqslant M_{2}$ iff $\widetilde{M_{1}} \leqslant \widetilde{M_{2}}$.

Proof. $(\Rightarrow)$ Suppose that $M_{1} \leqslant M_{2}$. Let $X_{i} \in \mathbb{H}$ with $i=1, \ldots n$ and $m=\min \left(\# X_{1}, \ldots\right.$,\#踟). Then by definition of $\leqslant \mathbb{H}$, it holds that

$$
\widetilde{M_{1}}\left(X_{1}, \ldots, X_{n}\right)=\left\{M_{1}\left(\sigma_{X_{1}}(i), \ldots, \sigma_{X_{n}}(i)\right): i=1, \ldots, m\right\} \leqslant{ }_{\mathbb{H}}\left\{M_{2}\left(\sigma_{X_{1}}(i), \ldots, \sigma_{X_{n}}(i)\right): i=1, \ldots, m\right\}=\widetilde{M_{2}}\left(X_{1}, \ldots, X_{n}\right) .
$$

$(\Leftarrow)$ If $\widetilde{M_{1}} \leqslant{ }_{\mathbb{H}} \widetilde{M_{2}}$, then trivially for each $x_{i} \in[0,1]$, with $i=1, \ldots, n$, then

$$
\left\{M_{1}\left(x_{1}, \ldots, x_{n}\right)\right\}=\widetilde{M_{1}}\left(\left\{x_{1}\right\} \ldots,\left\{x_{n}\right\}\right) \leqslant \widetilde{\mathbb{M}} \widetilde{M}_{2}\left(\left\{x_{1}\right\} \ldots,\left\{x_{n}\right\}\right)=\left\{M_{2}\left(x_{1}, \ldots, x_{n}\right)\right\}
$$

Therefore, by definition of $\leqslant_{\mathbb{H}}, M_{1}\left(x_{1}, \ldots, x_{n}\right) \leqslant M_{2}\left(x_{1}, \ldots, x_{n}\right)$.

Corollary 1. Let $T:[0,1]^{2} \rightarrow[0,1]$. Then $\widetilde{T}$ is an $\llbracket-t$-subnorm iff $T$ is a $\llbracket-t$-subnorm.

Proof. Straightforward from Theorem 2 and Lemma 1.

Corollary 2. Let $T:[0,1]^{2} \rightarrow[0,1]$. Then $\widehat{T}$ is an $\mathbb{\|}-t$-norm iff $T$ is a t-norm.

Proof. Straightforward from Theorem 3.

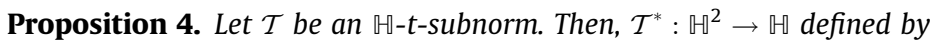

$$
\mathcal{T}^{*}(X, Y)= \begin{cases}\mathcal{T}(X, Y,) & \text { if } X \vee Y \neq \mathbf{1} ; \\ X \wedge Y, & \text { otherwise. }\end{cases}
$$

Proof. Straightforward.

Corollary 3. If $T$ is a t-subnorm then $\widetilde{T}^{*}$ is a $\llbracket-t-n o r m$.

Proof. Straightforward from Corollary 1 and Proposition 4.

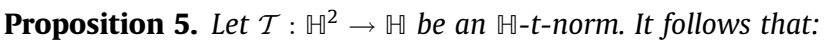

(i) If $\mathcal{T}$ verifies the cancellation law then it is strictly isotone;

(ii) if $\mathcal{T}$ is strictly isotonic then it has only trivial idempotent elements;

(iii) if $\mathcal{T}$ is strictly monotone then it has no divisors of zero.

Proof. Let $\mathcal{T}: \mathbb{H}^{2} \rightarrow \mathbb{U}$ be an $\mathbb{\sharp}$-t-norm, and $X_{1}, X_{2}, X_{3} \in \mathbb{U}$.

(i) If $X \neq \mathbf{0}$ and $Y<Z$ then by isotonic $\mathcal{T}(X, Y) \leqslant \mathcal{T}(X, Z)$. If $\mathcal{T}$ satisfies the cancellation law and $\mathcal{T}(X, Y)=\mathcal{T}(X, Z)$, then $X=\mathbf{0}$ or $X=Z$, which is a contradiction. Therefore, $\mathcal{T}$ is strictly isotonic. 
(ii) The existence of neutral element $\mathbf{1}$ and the strict monotonicity of $\mathcal{T}$ implies that $\mathcal{T}(X, X)<\mathcal{T}(X, \mathbf{1})=X$, for all $X \in \mathbb{U} \backslash\{\mathbf{0}, \mathbf{1}\}$. Therefore, $\mathcal{T}$ has only trivial idempotent elements.

(iii) The assumption that $A$ is a divisor of zero, i.e., $\mathcal{T}(A, X)=\mathbf{0}$ for some $X \in \mathbb{U} \backslash\{\mathbf{0}, \mathbf{1}\}$ implies that $\frac{X}{n}=\left\{\frac{x}{n}: x \in X\right\} \in \mathbb{U}$ is such that $\mathcal{T}(A, X)=\mathcal{T}\left(A, \frac{X}{n}\right)=\mathbf{0}$. This is a contradiction, since it violates the strict monotonicity of $\mathcal{T}$.

Therefore, Proposition 5 is verified.

\section{Corollary 4}

(iv) if $\mathcal{T}$ verifies the cancellation law, it has only trivial idempotent elements;

(v) if $\mathcal{T}$ verifies the cancellation law, it has no divisors of zero.

Proof. Straightforward from Proposition 5.

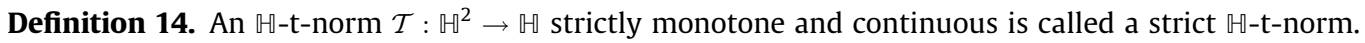

Proposition 6. Let $\mathcal{T}: \mathbb{H}^{2} \rightarrow \mathbb{W}$ be an $\mathbb{-}-t-n o r m$. It follows that:

(vi) if $\mathcal{T}$ is strict then it is strictly monotone;

(vii) if $\mathcal{T}$ is strict then it verifies the Archimedean property;

(viii) if $\mathcal{T}$ is strict then it verifies the cancellation law;

(ix) if $\mathcal{T}$ verifies the Archimedean property, it has only trivial idempotent elements.

Proof. Let $\mathcal{T}: \mathbb{\boxplus}^{2} \rightarrow \mathbb{}$ be an $\mathbb{-}-\mathrm{t}-$ norm.

(vi) Follows from Definition 14.

(vii) If $\mathcal{T}$ is a strictly monotone, by assertion (ii), it has only trivial idempotent elements. Then, it follows that, for all $X, Y \in \mathbb{U} \backslash\{\mathbf{0}, \mathbf{1}\}, \mathcal{T}(X, X)<\mathcal{T}(X, \mathbf{1})=X$. And so, it is clear that there exist at most finitely many integer $n$ with $\mathcal{T}^{n}(X)<_{\mathbb{H}} Y$, i.e. $\mathcal{T}$ is also Archimedean.

(viii) Follows immediately from assertions (i) and (vi).

(ix) In order to prove assertion (ix), let $\mathcal{T}$ be an $\mathbb{-}$-t-norm which verifies the Archimedean property. Then, for each $X \in \mathbb{U} \backslash\{\mathbf{0}, \mathbf{1}\}, \mathcal{T}(X, X)<_{\mathbb{H}} X$. So, no $X \in \mathbb{U} \backslash\{\mathbf{0}, \mathbf{1}\}$ is an idempotent element.

Therefore, Proposition 6 is verified.

Fig. 1 summarizes the assertions in Propositions 5 and 6, denoting by $\mathcal{C}(Z D), \mathcal{C}(S M), \mathcal{C}(I E), \mathcal{C}(C L), \mathcal{C}(S)$ and $\mathcal{C}(A P)$ to the classes of $\mathbb{H}$-t-norms which satisfy the properties $M 4, M 6, M 5-a, M 11$, and $M 10$.

\section{6. $H$-automorphisms acting on typical hesitant aggregation functions}

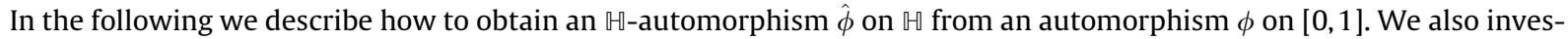
tigate two relevant aspects in Theorems 4 and 5: the action of an $\mathbb{U}$-automorphism on a binary THAF $\mathcal{T}$ and the preservation of its main properties in the class of $\mathbb{H}-\mathrm{t}$-norms.

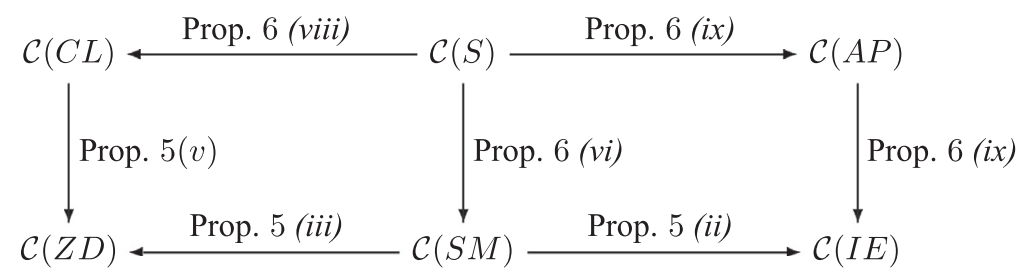

Fig. 1. Commutative diagram of $\mathbb{u - t}-\mathrm{t}$-norms. 
Definition 15. A function $\Phi: \mathbb{U} \rightarrow \mathbb{U}$ is an $\mathbb{-}$-automorphism if it is bijective and, for each $X, Y \in \mathbb{H}, X \leqslant \mathbb{H} Y$ iff $\Phi(X) \leqslant \Phi(Y)$.

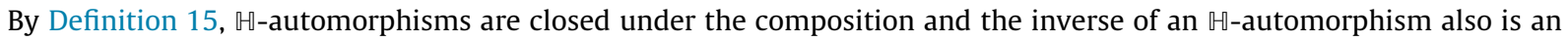
$\mathbb{H}$-automorphism:

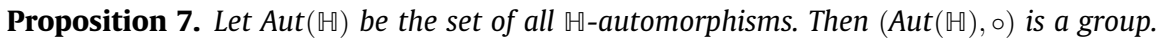

Proof. Trivially, the composition of bijective and isotonic functions on a set, in this case $\mathbb{H}$, is also bijective and isotonic. Therefore, $\mathbb{-}$-automorphisms are closed under composition. The composition of functions is always associative and the

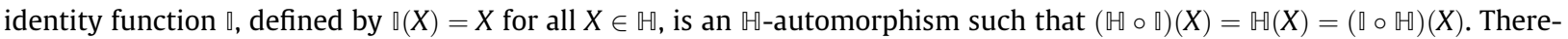
fore, $($ Aut $(\mathbb{H}), \circ)$ has a neutral element. Thus, from the definition of $\mathbb{H}$-automorphism, the inverse of an $\mathbb{H}$-automorphism also

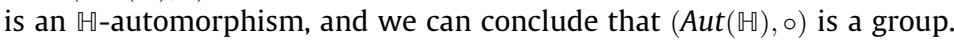

Lemma 2. Let $\Phi: \mathbb{W} \rightarrow \mathbb{W}$ be an $\mathbb{M}$-automorphism. Then for each $X \in \mathbb{U}$ such that $\# X \geqslant 2$, \# $\Phi(X) \geqslant 2$.

Proof. By the definition of $\wedge$, for every $X_{i} \in \mathbb{H}$ with $i \in \mathbb{N}_{n}$, if $\# \sum_{i=1}^{n} X_{i}=1$ then $X_{j}=\sum_{i=1}^{n} X_{i}$ for some $j \in \mathbb{N}_{n}$. Also, observe that $\mathbb{H}$-automorphisms are isomorphisms over the lattice $\left(\mathbb{H}, \leqslant_{\mathbb{H}}\right)$ and so $\Phi\left(\sum_{i=1}^{n} X_{i}\right)=\sum_{i=1}^{n} \Phi\left(X_{i}\right)$. Suppose that for some $X \in \mathbb{H}$ with $n=\# X \geqslant 2, \Phi(X)=\{y\}$ for some $y \in[0,1]$. Without loss of generality we can consider that $\sigma_{X}(1) \neq 0$. Then defining $X_{i}=\left\{\frac{\sigma_{X}(1)}{j}: j<i\right\} \cup\left\{\sigma_{X}(i)\right\} \cup\left\{\sigma_{X}(i)+\frac{\sigma_{X}(i+1)-\sigma_{X}(i)}{j}: i<j \leqslant n\right\}$, clearly from definition of $\vee$ for the lattice $(\mathbb{H}, \leqslant \mathbb{H}), X=\sum_{i=1}^{n}$ and $X \neq X_{i}$. So, $\{y\}=\Phi(X)=\Phi\left(\sum_{i=1}^{n} X_{i}\right)=\sum_{i=1}^{n} \Phi\left(X_{i}\right)$ and therefore, $\Phi\left(X_{i}\right)=\{y\}$ which is in contradiction with the bijectivity of $\Phi$.

Proposition 8. Let $\hat{\phi}: \mathbb{H} \rightarrow \mathbb{U}$ be an $\mathbb{H}$-automorphism. Then for each $x \in[0,1]$ there exists $y \in[0,1]$ such that $\hat{\phi}(\{x\})=\{y\}$.

Proof. Suppose that for some $x \in[0,1]$, \# $\Phi(\{x\}) \geqslant 2$. Then, by Lemma $2, \#\{x\}=\# \Phi^{-1}(\Phi(\{x\})) \geqslant 2$ which is an absurd.

Theorem 4. $\hat{\phi}: \mathbb{H} \rightarrow \mathbb{W}$ is an $\mathbb{H}$-automorphism iff there exists an automorphism $\phi:[0,1] \rightarrow[0,1]$ such that, for all $X \in \mathbb{U}$, it follows that $\widehat{\phi}(X)=\{\phi(x): x \in X\}$.

Proof. $(\Rightarrow)$ Let $\hat{\phi}: \mathbb{H} \rightarrow \mathbb{U}$ be an $\mathbb{U}$-automorphism and define $\phi:[0,1] \rightarrow[0,1]$ by $\phi(x)=\sigma_{\hat{\phi}(\{x\})}(1)$. By Proposition 8 , it is clear that $\phi$ is an automorphism and that $\hat{\phi}$ and $\phi$ satisfy Eq. (18).

$(\Leftarrow)$ Let $\phi:[0,1] \rightarrow[0,1]$ be an automorphism. First, suppose $X, Y \in \mathbb{U}$ such that $X \neq Y$. We have two cases, there exists $\bar{x} \in X-Y$ or $\bar{y} \in Y-X$. Based on the automorphism $\phi$, we have that: (i) $\phi(\bar{x}) \neq \phi(y)$, for all $y \in Y$; or (ii) $\phi(x) \neq \phi(\bar{y})$, for all $x \in X$. Both cases imply $\{\phi(x): x \in X\} \neq\{\phi(y): y \in Y\}$, which means that $\hat{\phi}(X) \neq \hat{\phi}(Y)$. Therefore $\hat{\phi}$ is an injective function over $\mathbb{H}$. In addition, since $\phi$ is an automorphism, for all $Y \in \mathbb{H}$, if $X=\left\{\phi^{-1}(y): y \in Y\right\}$ then $\hat{\phi}(X)=\left\{\left(\phi \circ \phi^{-1}\right)(y): y \in Y\right\}=Y$. So, $\hat{\phi}$ is a bijective function over $\mathbb{H}$. Now, considering the $\alpha$-normalization and $m=\min (\# X$,\#Y), for $X, Y \in \mathbb{M}$, suppose that $X \leqslant \mathbb{\sharp} Y$. One of the two next cases is held:

(i) $\alpha(X, \# Y)<_{\mathbb{H}^{(m)}} \alpha(Y, \# X)$ : since $\phi$ is a non-decreasing function and denoting $\alpha(X, m)$ by $\widetilde{X}$ and $\alpha(Y, m)$ by $\widetilde{Y}$, for each $1 \leqslant i \leqslant m$, we have that $\phi\left(\sigma_{\widetilde{X}}(i)\right) \leqslant \phi\left(\sigma_{\widetilde{Y}}(i)\right)$. Thus, we obtain that $\hat{\phi}(\widetilde{X})=\left\{\phi\left(\sigma_{\widetilde{X}}(i)\right): 1 \leqslant i \leqslant m\right\} \leqslant \mathbb{t}\left\{\phi\left(\sigma_{\widetilde{Y}}(i)\right)\right.$ : $1 \leqslant i \leqslant m\}=\hat{\phi}(\widetilde{Y})$, implying that $\hat{\phi}(X) \leqslant 甘 \hat{\phi}(Y)$.

(ii) $\alpha(X, \# Y)=\alpha(Y, \# X)$ and $\# Y \leqslant \# X$ : for each $1 \leqslant i \leqslant m$, we have that $\phi\left(\sigma_{\widetilde{X}}(i)\right)=\phi(\sigma \widetilde{\sim}(i))$. Thus, we obtain that $\hat{\phi}(\widetilde{X})=\left\{\phi\left(\sigma_{\widetilde{X}}(i)\right): 1 \leqslant i \leqslant m\right\} \leqslant_{\mathbb{H}}\left\{\phi\left(\sigma_{\widetilde{X}}(i)\right): 1 \leqslant i \leqslant m\right\}=\hat{\phi}(\widetilde{Y})$, which also means that $\hat{\phi}(X) \leqslant \mathbb{H}(Y)$.

In both cases, $\hat{\phi}$ is a non-decreasing function on $\mathbb{H}$.

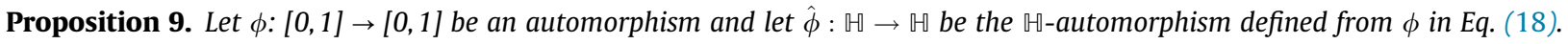
Then it holds that

$$
\widehat{\phi^{-1}}=\hat{\phi}^{-1} \text {. }
$$

Proof. Let $X \in \mathbb{M}$. Then $\hat{\phi}\left(\widehat{\phi^{-1}}(X)\right)=\hat{\phi}\left(\left\{\phi^{-1}(x): x \in X\right\}=\left\{\phi\left(\phi^{-1}(x)\right): x \in X\right\}=X\right.$ So, $\widehat{\phi^{-1}}$ is an inverse of $\hat{\phi}$.

Given a function $F: \mathbb{U}^{n} \rightarrow \mathbb{U}$ and an $\mathbb{U}$-automorphism $\Phi$, the action of $\Phi$ on $F$ is the function $F^{\Phi}: \mathbb{U}^{n} \rightarrow \mathbb{U}$ called conjugate function of $F$ and defined by

$$
F^{\Phi}\left(X_{1}, \ldots, X_{n}\right)=\Phi^{-1}\left(F\left(\Phi\left(X_{1}\right), \ldots, \Phi\left(X_{n}\right)\right)\right) .
$$


Theorem 5. Let $\mathcal{T}: \mathbb{H}^{2} \rightarrow \mathbb{H}$ be a binary THAF and $\Phi: \mathbb{H} \rightarrow \mathbb{H}$ be an $\mathbb{H}$-automorphism defined by $\phi$ in Eq. (18). It follows that:

(i) $\mathcal{T}^{\Phi}$ is an $\mathbb{H}$-t-norm iff $\mathcal{T}$ is an $\mathbb{H}$-t-norm;

(ii) $\mathcal{T}^{\Phi}$ has an annihilator element iff $\mathcal{T}$ has an annihilator element;

(iii) $\mathcal{T}^{\Phi}$ has divisors of zero iff $\mathcal{T}$ has divisors of zero;

(iv) $\mathcal{T}^{\Phi}$ is idempotent iff $\mathcal{T}$ is idempotent;

(v) $\mathcal{T}^{\Phi}$ is strictly isotonic iff $\mathcal{T}$ is strictly isotonic;

(vi) $\mathcal{T}^{\Phi}$ is Archimedean iff $\mathcal{T}$ is Archimedean;

(vii) $\mathcal{T}^{\Phi}$ verifies the cancellation law iff $\mathcal{T}$ verifies the cancellation law;

(viii) $\mathcal{T}^{\Phi}$ has a nilpotent element iff $\mathcal{T}$ has a nilpotent element;

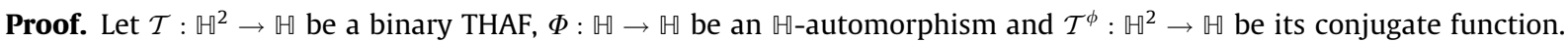

(i) If $\mathcal{T}$ is an $\mathbb{H}$-t-norm, for all $X_{1}, X_{2}, X_{3} \in \mathbb{H}$, it follows that:

(1) $\mathcal{T}^{\Phi}$ is symmetric:

$\mathcal{T}^{\Phi}\left(X_{1}, X_{2}\right)=\Phi^{-1}\left(\mathcal{T}\left(\Phi\left(X_{1}\right), \Phi\left(X_{2}\right)\right)\right)=\Phi^{-1}\left(\mathcal{T}\left(\Phi\left(X_{2}\right), \Phi\left(X_{1}\right)\right)\right)=\mathcal{T}^{\Phi}\left(X_{2}, X_{1}\right)$

(2) $\mathcal{T}^{\Phi}$ is associative:

$$
\begin{aligned}
\mathcal{T}^{\Phi}\left(\mathcal{T}^{\Phi}\left(X_{1}, X_{2}\right), X_{3}\right) & =\mathcal{T}^{\Phi}\left(\Phi^{-1}\left(\mathcal{T}\left(\Phi\left(X_{1}\right), \Phi\left(X_{2}\right)\right)\right), X_{3}\right)=\Phi^{-1}\left(\mathcal{T}\left(\mathcal{T}\left(\Phi\left(X_{1}\right), \Phi\left(X_{2}\right)\right), \Phi\left(X_{3}\right)\right)\right. \\
& =\Phi^{-1}\left(\mathcal{T}\left(\Phi\left(X_{1}\right), \mathcal{T}\left(\Phi\left(X_{2}\right)\right), \Phi\left(X_{3}\right)\right)\right)=\mathcal{T}^{\Phi}\left(X_{1}, \mathcal{T}^{\Phi}\left(X_{2}, X_{3}\right)\right)
\end{aligned}
$$

(3) $\mathcal{T}^{\Phi}$ has 1 as neutral element:

$$
\mathcal{T}^{\Phi}\left(X_{1}, \mathbf{1}\right)=\Phi^{-1}\left(\mathcal{T}\left(\Phi\left(X_{1}\right), \Phi(\mathbf{1})\right)\right)=\Phi^{-1}\left(\mathcal{T}\left(\Phi\left(X_{1}\right), \mathbf{1}\right)\right)=\Phi^{-1}\left(\Phi\left(X_{1}\right)\right)=X_{1}
$$

(ii) If $\mathcal{T}$ has an annihilator element $A \in \mathbb{H}$ we get that

$$
\mathcal{T}^{\Phi}\left(X, \Phi^{-1}(A)\right)=\Phi^{-1}\left(\mathcal{T}\left(\Phi(X), \Phi\left(\Phi^{-1}(A)\right)\right)\right)=\Phi^{-1}\left(\mathcal{T}(\Phi(X), A)=\Phi^{-1}(A) .\right.
$$

Thus, $\Phi^{-1}(A)$ is an annihilator of $\mathcal{T}^{\Phi}$.

(iii) If $X, Y \in \mathbb{U} \backslash\{\mathbf{0}, \mathbf{1}\}$ are such that $\mathcal{T}(X, Y)=\mathbf{0}$, then $\mathcal{T}^{\Phi}\left(\Phi^{-1}(X), \Phi^{-1}(Y)\right)=\Phi^{-1}(\mathcal{T}(X, Y))=\mathbf{0}$. Since, $\Phi^{-1}(X) \neq \mathbf{0}$ and $\Phi^{-1}(-$ $Y) \neq \mathbf{0}$, then $\Phi^{-1}(X)$ is a divisor of zero of $\mathcal{T}^{\Phi}$.

(iv) If $\mathcal{T}$ is idempotent and $\Phi$ is an $\mathbb{-}$-automorphism, property $M 5$ is fulfilled by the conjugate of $\mathcal{T}$ :

$\mathcal{T}^{\Phi}(X, X)=\Phi^{-1}(\mathcal{T}(\Phi(X), \Phi(X)))=\Phi^{-1}(\Phi(X))=X$, for all $\Phi(X) \in \mathbb{H}$.

(v) Let $\mathcal{T}$ be strictly isotonic function. In this case we have the following: $\mathcal{T}^{\Phi}(X, Y)=$ $\Phi^{-1}(\mathcal{T}(\Phi(X), \Phi(Y)))<_{\sharp} \Phi^{-1}(\mathcal{T}(\Phi(X), \Phi(Z)))=\mathcal{T}^{\Phi}(X, Z)$, whenever $X \neq \mathbf{0}$ and $Y<_{\sharp} Z$. So, $\mathcal{T}^{\Phi}$ is also a strictly isotonic function.

(vi) Let the $\mathbb{H}$-t-norm $\mathcal{T}$ be Archimedean and $\Phi$ is an $\mathbb{H}$-automorphism. Then, for each $X, Y \in \mathbb{U} \backslash\{\mathbf{1}, \mathbf{0}\}$, we have that $\Phi(X), \Phi(Y) \in \mathbb{U} \backslash\{\mathbf{1}, \mathbf{0}\}$. Therefore, because $\mathcal{T}$ is Archimedean there exists $n \in \mathbb{N}$ such that $\mathcal{T}^{n}(\Phi(X))<_{\mathbb{H}} \Phi(Y)$ and so, $\left(\mathcal{T}^{\Phi}\right)^{n}(X)=\Phi^{-1}\left(\mathcal{T}^{n}(\Phi(X))\right)<_{\mathbb{H}} Y$, i.e. $\mathcal{T}^{\Phi}$ also is Archimedean.

(vii) Let $\mathcal{T}$ be a $\mathbb{U}$-t-norm verifying the cancellation law, whereas $\Phi$ is an $\mathbb{H}$-automorphism. If $\mathcal{T}^{\Phi}(X, Y)=\mathcal{T}^{\Phi}(X, Z)$, then because $\Phi^{-1}$ is bijective, $\mathcal{T}(\Phi(X), \Phi(Y))=\mathcal{T}(\Phi(X), \Phi(Z))$. Since $\mathcal{T}$ verifies the cancellation law, we have that $\Phi(X)=\mathbf{0}$ implies that $X=\mathbf{0}$ or $\Phi(Y)=\Phi(Z)$ and, in this case, we can conclude that $Y=Z$.

(viii) Let $T$ be a nilpotent element of $\mathcal{T}$. So, $T \in \mathbb{U} \backslash\{\mathbf{0}, \mathbf{1}\}$ and based on the $\mathbb{M}$-automorphism $\Phi$, it also means that $\Phi^{-1}(T) \in \mathbb{U} \backslash\{\mathbf{0}, \mathbf{1}\}$. So, there exists $n \in \mathbb{N}$ such that $\mathcal{T}^{n}(T)=\mathbf{0}$ and it follows that $\left(\mathcal{T}^{\Phi}\right)^{n}\left(\Phi^{-1}(T)\right)=\Phi^{-1}\left(\mathcal{T}^{n}\left(\Phi\left(\Phi^{-1}(T)\right)\right)=\Phi^{-1}\left(\mathcal{T}^{n}(T)\right)=\Phi^{-1}(\mathbf{0})=\mathbf{0}\right.$. Therefore, it holds that $\mathcal{T}^{\Phi}\left(\Phi^{-1}(T)\right)=\mathbf{0}$. This shows $\Phi^{-1}(T)$ is a nilpotent element of $\mathcal{T}^{\Phi}$.

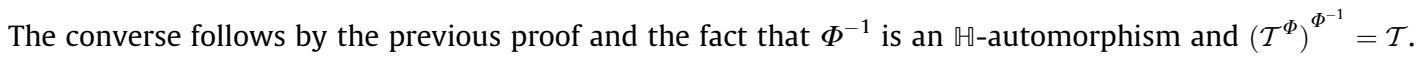

\section{Final remarks}

In this paper we have studied the THAFs defined over the set of all typical hesitant fuzzy sets by considering two methodologies, both compatible with the partial order $\leqslant_{\mathbb{H}}$, whose definition is based on $\alpha$-normalization and $\sigma$-permutation. The main advantage of such methods against other similar methods is the intuitive way to deal with the monotonicity of THAFs. This intuitive way can be extended to construct ordered aggregation operators for THFEs, as requirements for modelling and grouping the information obtained from different sources. 
Together with the main properties of THAFs extended from AFs, we also investigate their conjugate functions obtained by action of $\mathbb{\square}$-automorphisms. In particular, the class of $\mathbb{\square}$-t-norms was considered and the conditions under which main properties of such aggregation functions are preserved by their conjugate functions were also presented.

Further work intends to investigate other theoretical aspects related to typical hesitant fuzzy t-conorms ( $\mathbb{-}$ - $\mathrm{t}$-conorms)

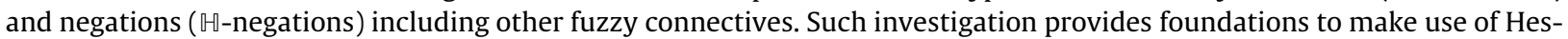
itant Fuzzy Logic in several applied fields, such as decision making.

\section{Acknowledgments}

This work is supported by the Brazilian funding agencies CNPq (Ed. PQ and universal, under the Process Numbers 307681/ 2012-2 and 480832/2011-0, respectively) and FAPERGS (Ed. PqG 06/2010, under the process number 11/1520-1) and by the project TIN2010-15055 of the Spanish Ministry of Science.

\section{References}

[1] K.H. Atanassov, Intuitionistic fuzzy sets, Fuzzy Sets and Systems 20 (1) (1986) 87-96.

[2] K.H. Atanassov, G. Gargov, Elements of intuitionistic fuzzy logic - Part I, Fuzzy Sets and Systems 95 (1) (1989) $39-52$.

[3] K.H. Atanassov, G. Gargov, Interval valued intuitionistic fuzzy sets, Fuzzy Sets and Systems 31 (3) (1989) $343-349$.

[4] K.H. Atanassov, Intuitionistic Fuzzy Sets, Theory and Applications (Studies in Fuzziness and Soft Computing), Physica-Verlag, Heidelberg, 1999.

[5] B. Bedregal, On interval fuzzy negations, Fuzzy Sets and Systems 161 (17) (2010) 2290-2313.

[6] B. Bedregal, G. Dimuro, R. Santiago, R. Reiser, On interval fuzzy S-implications, Information Sciences 180 (8) (2010) $1373-1389$.

[7] B. Bedregal, R. Santiago, Some continuity notions for interval functions and interval representation, Computational and Applied Mathematics. doi:10.1007/s40314-013-0049-z.

[8] P. Burillo, H. Bustince, Vague sets are intuitionistic fuzzy sets, Fuzzy Sets and Systems 79 (3) (1996) $403-405$.

[9] H. Bustince, P. Burillo, F. Soria, Automorphism, negations and implication operators, Fuzzy Sets and Systems 134 (2003) $209-229$.

[10] H. Bustince, J. Montero, R. Mesiar, Migrativity of aggregation operators, Fuzzy Sets and Systems 160 (6) (2009) $766-777$.

[11] T. Calvo, A. Kolesárová, M. Komorníkova, R. Mesiar, Aggregation operators: properties, classes and construction methods, in: Aggregation Operators New Trends and Applications, Physica-Verlag, Heidelberg, 2002.

[12] N. Chen, Z. Xu, M. Xia, Interval-valued hesitant preference relations and their applications to group decision making, Knowledge-Based Systems 37 (2013) 357-365.

[13] N. Chen, Z. Xu, M. Xia, Correlation coefficients of hesitant fuzzy sets and their applications to clustering analysis, Applied Mathematical Modelling 37 (4) (2013) 2197-2211.

[14] G. Deschrijver, C. Cornelis, Representability in interval-valued fuzzy set theory, International Journal of Uncertainty, Fuzziness and Knowledge-Based Systems 15 (3) (2007) 345-361.

[15] G. Deschrijver, E. Kerre, Aggregation operators in interval-valued fuzzy and Atanassov's intuitionistic fuzzy set theory, Fuzzy Sets and their Extensions: Representation, Aggregation and Models (2008) 83-203.

[16] G.P. Dimuro, B.C. Bedregal, R.H.N. Santiago, R.H.S. Reiser, Interval additive generators of interval t-norms and interval t-conorms, Information Sciences 181 (18) (2011) 3898-3916.

[17] D. Dubois, J.L. Koning, Social choice axioms for fuzzy set aggregation, Fuzzy Sets and Systems 58 (3) (1991) $339-342$.

[18] D. Dubois, W. Ostasiewicz, H. Prade, Fuzzy Sets: History and Basic Notions in Fundamentals of Fuzzy Sets, Kluwer, Boston, 2000.

[19] D. Dubois, H. Prade, Fundamentals of Fuzzy Sets, The Handbooks of Fuzzy Sets Series, Kluwer, Boston, 2000.

[20] B. Farhadinia, A novel method of ranking hesitant fuzzy values for multiple attribute decision-making problems, International Journal of Intelligent Systems 28 (8) (2013) 752-767.

[21] B. Farhadinia, Information measures for hesitant fuzzy sets and interval-valued hesitant fuzzy sets, Information Sciences 240 (2013) $129-144$.

[22] W.L. Gau, D.J. Buehrer, Vague sets, IEEE Transactions on Systems, Man and Cybernetics 23 (2) (1993) $610-614$.

[23] E.P. Klement, R. Mesiar, E. Pap, Triangular norms, trends in logic, Studia Logica Library, vol. 8, Kluwer Academic Publishers., Dordrecht, 2000.

[24] R. Mesiar, A. Kolesárová, T. Calvo, M. Komorníková, A review of aggregation functions, in: H. Bustince et al. (Eds.), Fuzzy Sets and their Extensions: Representation, Aggregation and Models, Studies in Fuzziness and Soft Computing, vol. 220, Springer, 2008, pp. 121-144.

[25] R. Mesiar, M. Komorníková, Aggregation functions on bounded posets, in: C. Cornelis, G. Deschrijver, M. Nachtegael, S. Schockaert, Y. Shi (Eds.), 35 Years of fuzzy set theory - celebratory volume dedicated to the retirement of Etienne Kerre, Studies in Fuzziness and Soft Computing, vol. 261, Springer, 2011, pp. 3-18.

[26] G. Qian, H. Wang, X. Feng, Generalized hesitant fuzzy sets and their application in decision support system, Knowledge-Based Systems 37 (2013) $357-$ 365.

[27] R.H.S. Reiser, B. Bedregal, Interval-valued intuitionistic fuzzy implications - construction, properties and representability, Information Sciences 248 (2013) 68-88.

[28] R.M. Rodríguez, L. Martínez, F. Herrera, Hesitant fuzzy linguistic term sets for decision making, IEEE Transactions on Fuzzy Systems 20 (1) (2012) 109119.

[29] R.M. Rodríguez, L. Martínez, F. Herrera, A group decision making model dealing with comparative linguistic expressions based on hesitant fuzzy linguistic term sets, Information Sciences 241 (2013) 28-42.

[30] R. Sambuc, Fonctions $\phi$-floues. Application l'aide au diagnostic en pathologie thyroidienne, PhD thesis, Univ. Marseille, Marseille, 1975.

[31] R.H.N. Santiago, B. Bedregal, B.M. Acióly, Formal aspects of correctness and optimality of interval computations, Formal Aspects of Computing 18 (2) (2006) 231-243.

[32] B. Schweizer, A. Sklar, Probabilistic Metric Spaces, North-Holland, Amsterdam, 1983.

[33] V. Torra, Y. Narukawa, On hesitant fuzzy sets and decision, in: Proceedings of FUZZ-IEEE, 2009, pp. 1378-1382.

[34] V. Torra, Hesitant fuzzy sets, International Journal of Intelligent Systems 25 (2010) 529-539.

[35] G. Wei, Hesitant fuzzy prioritized operators and their application to multiple attribute decision making, International Journal of Uncertainty, Fuzziness and Knowledge-Based Systems 31 (2012) 176-182.

[36] G. Wei, X. Zhao, R. Lin, Some hesitant interval-valued aggregation operators and their applications to multiple attribute decision making, KnowledgeBased Systems 46 (2013) 43-53.

[37] M. Xia, Z. Xu, Hesitant fuzzy information aggregation in decision making, International Journal of Approximate Reasoning 52 (3) (2011) 395-407.

[38] M. Xia, Z. Xu, N. Chen, Induced aggregation under confidence levels, International Journal of Uncertainty, Fuzziness and Knowledge-Based Systems 19 (2) (2011) 201-227.

[39] M. Xia, Z. Xu, N. Chen, Some hesitant fuzzy aggregation operators with their application in group decision making, Group Decision and Negotiation 22 (2) (2013) 259-279.

[40] Z. Xu, M. Xia, On distance and correlation measures of hesitant fuzzy information, International Journal of Intelligent Systems 26 (2011) $410-425$. 
[41] Z. Xu, M. Xia, Distance and similarity measures for hesitant fuzzy sets, Information Sciences 181 (11) (2011) 2128-2138.

[42] Z. Xu, M. Xia, Hesitant fuzzy entropy and cross-entropy and their use in multiattribute decision-making, Journal of Intelligent Systems 27 (2013) $799-$ 822.

[43] R.R. Yager, On ordered weighted averaging aggregation operators in multi-criteria decision making, IEEE Transactions on Systems, Man and Cybernetics 18 (1) (1988) 183-190.

[44] L.A. Zadeh, Fuzzy sets, Information Control 8 (1965) 338-353.

[45] L.A. Zadeh, The concept of a linguistic variable and its application to approximate reasoning - I, Information Sciences 8 (3) (1975) $199-249$.

[46] L.A. Zadeh, Fuzzy sets as a basis for a theory of possibility, Fuzzy Sets and Systems 1 (1978) 3-28.

[47] Z. Zhang, Hesitant fuzzy power aggregation operators and their application to multiple attribute group decision making, Information Sciences 234 (10) (2013) 150-181.

[48] B. Zhu, Z. Xu, M. Xia, Hesitant fuzzy geometric Bonferroni means, Information Sciences 205 (1) (2012) 72-85. 